\title{
Aux origines du chaos. Analyse du concept d'Isfet dans la Prophétie de Néferty
}

Par

\section{Laura Parys}

Université catholique de Louvain

$\mathrm{L}$

a Prophétie de Néferty est un texte littéraire de l'Égypte ancienne ${ }^{1}$, qui est préservé intégralement au verso d'un papyrus attribué à la XVIII ${ }^{\text {e }}$ dynastie et conservé au musée de Saint-Pétersbourg (pErmitage $1116 \mathrm{~B})^{2}$. Il existe des témoins additionnels, à savoir deux tablettes de bois (Londres BM 5647 et Caire CG 25224), vingt-quatre ostraca ${ }^{3}$ et trois graffiti inscrits dans la tombe N $13.1 \mathrm{~d}^{\prime}$ Assiout $^{4}$. Ils sont tous attribués aux XVIII ${ }^{\mathrm{e}}, \mathrm{XIX}^{\mathrm{e}}$ et $\mathrm{XX}^{\mathrm{e}}$ dynasties. L'édition en usage de nos jours reste celle de Helck, publiée en 1970, qui inclut une transcription hiéroglyphique, une traduction et des commentaires.

Le récit a pour cadre chronologique le règne de Snéfrou, fondateur de la $\mathrm{IV}^{\mathrm{e}}$ dynastie. Celui-ci convoque en son palais le ritualiste Néferty, afin qu'il lui dévoile l'avenir. Le sage commence par une longue lamentation sur les fléaux qui s'abattront sur l'Égypte, avant de prédire la venue d'un roi sauveur nommé Amény et originaire de la région méridionale. Les

\footnotetext{
${ }^{1}$ Plusieurs commentateurs estiment que le terme «prophétie » habituellement utilisé pour désigner l'œuvre est inadéquat. Il serait préférable de parler de «prédiction », afin d'éviter toute confusion avec les prophéties bibliques : cf. BLUMENTHAL 1982, p. 17 ; WEIPPERT 1988, p. 287-319 ; SHUPAK 2006, p. 133-144.

${ }^{2}$ Photographies : GolÉNISCHEFF 1913, pl. XXIII-XXV.

${ }^{3}$ MATHIEU 1993, p. 343, n. 43, en fournit la liste la plus récente.

${ }^{4}$ VERHOEVEN 2013, p. 143, n. 6a-6c.
} 
circonstances de l'avènement d'Amény et la mention des « Murs du Souverain » ont poussé la plupart des commentateurs à l'identifier à Amenemhat I ${ }^{\mathrm{er}}$, fondateur de la XII $\mathrm{Xynastie}^{\mathrm{e}}$. Concernant les troubles décrits par Néferty, ils furent interprétés comme se référant à la Première Période intermédiaire ${ }^{6}$, voire à la période d'interrègne précédant l'avènement d'Amenemhat $\mathrm{I}^{\text {er7 }}$. Le texte fut longtemps considéré comme une œuvre de propagande en faveur de ce roi, jusqu'à ce que l'étude linguistique de Stauder infirme cette hypothèse en situant la composition du récit entre le milieu de la XIII ${ }^{\mathrm{e}}$ et le début de la XVIII ${ }^{\mathrm{e}}$ dynastie $^{8}$.

L'intérêt du récit de la Prophétie de Néferty réside dans ses descriptions du bouleversement de la société égyptienne avant l'avènement du roi Amény. Il s'agit d'une illustration du concept égyptien d'Isfet, qui s'oppose à la Maât (ordre cosmique et social) et désigne les divers aspects du chaos, notamment les troubles politiques et sociaux ${ }^{9}$. Les conditions qui ont favorisé, à un moment donné, la perte de la Maât au profit d'Isfet sont rarement interprétées par les commentateurs. Selon Lefèbvre et Posener, la cause principale du démantèlement du pays serait l'intrusion étrangère mentionnée dans le récit ${ }^{10}$. En revanche, Parkinson estime que Néferty n'expliciterait pas les origines du chaos, mais qu'il se contenterait d'énumérer les catastrophes d'ordre cosmique, politique et social, subies par le peuple égyptien à cette époque $^{11}$. Enfin, l'étude de Blumenthal ne propose pas d'interprétation globale de la Prophétie de Néferty, car elle considère sa structure trop désordonnée pour que l'on puisse y déceler un récit cohérent ${ }^{12}$. Elle argumente dès lors en faveur d'une composition hétérogène du texte, qui aurait subi des ajouts et des arrangements, à partir d'une ou de plusieurs versions préliminaires ${ }^{13}$.

En réalité, une analyse approfondie des thématiques abordées par Néferty dans sa prédiction permet de distinguer les causes et les conséquences des diverses calamités qu'il expose au roi Snéfrou. Afin de déterminer les origines du chaos dans le récit, nous proposerons une analyse comparative différentielle du concept d'Isfet au sein des deux principales sections du discours de Néferty : les « lamentations », qui illustrent le concept d'Isfet, et le « roi Amény », qui décrit la restauration de la Maât. Les résultats auxquels nous serons parvenue seront corroborés par une analyse intertextuelle, qui examinera les relations unissant la Prophétie de

\footnotetext{
${ }^{5}$ Notamment PoSEner 1956, p. 22-23. Par contre, RyHOLt 1990, p. 109, suggère une identification avec Amenemhat VI, voire avec Amenemhat III ou IV, alors que QUIRKE 2004, p. 135, propose « one or all of the four kings of the Twelfth Dynasty named Amenemhet, or a king of the Thirteenth Dynasty ». Récemment, GIEWEKEMEYER 2013, p. 345-353, a souligné qu'aucune identification claire d'Amény n'était possible, puisqu'il pourrait aussi bien s'agir d'Amenemhat Ir que de Montouhotep II ou d'Amosis.

${ }^{6}$ LefÈbVRe 1949, p. 92 ; Posener 1956, p. 44-47 ; LaLOUetTe 1984, p. 74.

${ }^{7}$ ERMAN 1923, p. 152 ; POSENER 1956, p. 44-47.

${ }^{8}$ STAUDER 2013, p. 511. Peu nombreuses sont les interprétations de la Prophétie de Néferty qui, tout en acceptant la référence à Amenemhat $\mathrm{I}^{\mathrm{er}}$ dans le personnage d'Amény, retiennent une composition du récit qui soit postérieure à la XII ${ }^{\mathrm{e}}$ dynastie. Cf. PARKINSON 2002, p. 304 ; GNIRS 2006, p. 243-253 ; PARYS 2017, p. 193194. 34.

${ }^{9}$ Concernant la notion d'Isfet, cf. ASSMANn 1994, p. 93-100 ; SMITH 1994, p. 67-88 ; FrandSEN 2000, p. 9-

${ }^{10}$ LEFÈBVRE 1949, p. 94 ; Posener 1956, p. 40.

${ }^{11}$ PARKINSON 2002, p. 197.

12 Blumenthal 1982, p. 12-13.

${ }^{13}$ Idem. Blumenthal estime que les commentaires sur la déchéance d'Héliopolis (XII, g) et sur la menace asiatique (VI, f - VIII, b) constitueraient des ajouts ultérieurs de l'auteur, en vue d'adapter le texte en fonction du message à transmettre.
} 
Néferty à d'autres textes égyptiens développant ce concept d'Isfet. Nous serons ainsi en mesure de proposer une interprétation novatrice de la Prophétie de Néferty, qui mettra en exergue l'homogénéité et la cohérence du récit.

\section{Analyse comparative du concept d'Isfet dans la Prophétie de Néferty}

\section{Les lamentations}

En guise d'introduction à son discours, le ritualiste Néferty incite son interlocuteur à prendre conscience du déclin du pays, en soulignant deux facteurs qui résument cet état : la destruction de la création et l'indifférence du peuple égyptien.

$(\mathrm{IV}, \mathrm{c}-\mathrm{V}$, a) La création est comme si elle n'avait jamais existé. Que Rê (re)commence à fonder le pays détruit dans sa totalité, (car) il ne restera plus rien : de ce qu'il avait instauré, il n'y aura (même) plus le noir de l'ongle. Ce pays sera détérioré, sans personne qui s'en souciera, personne qui (en) parlera et personne qui (le) pleurera.

Le sage Néferty détaille la destruction de la création dans la suite de son discours : à la question rhétorique «Comment sera ce pays?» $(\mathrm{V}, \mathrm{b})$, il répond en décrivant des perturbations atmosphériques (V, c - VI, e), une invasion des étrangers dans le Delta du Nil (VI, fVIII, d) et des bouleversements de la situation interne du pays (VIII, e - XII, g).

\section{Origine : perturbations atmosphériques}

Les perturbations atmosphériques que Néferty décrit sont au nombre de trois : un obscurcissement du soleil, une sécheresse due aux crues insuffisantes du Nil et une confrontation des vents $^{14}$.

(V, c-e) Le soleil sera voilé : il ne brillera plus, quand les gens (le) regarderont. Il est impossible que l'on vive quand le temps orageux (le) voile, si bien que tout homme sera engourdi (litt. sourd) [a] par manque de lui.

(VI, a-d) Les cours d'eau de l'Égypte seront vides: on traversera l'eau à pied et on cherchera de l'eau pour la flotte en vue de ses navigations. En effet, sa route se sera transformée en rive : alors que la rive sera devenue des flots, l'emplacement de l'eau sera ce qui était l'emplacement de la rive.

(VI, e) Le vent du Sud s'opposera au vent du Nord, si bien que le ciel ne sera plus (animé) par un vent unique.

[a] Le verbe idi signifie « être sourd» $(W b . \mathrm{I}, 151,13)$. Certains traducteurs ont gardé cette traduction littérale ${ }^{15}$, tandis que d'autres l'ont compris comme l'engourdissement des sens de la vue et de l'ouie ${ }^{16}$. Autres traductions : « dulled $»^{17} ;\left\langle\right.$ abasourdi $»^{18} ;\left\langle\right.$ darkened ${ }^{19}$.

\footnotetext{
${ }^{14}$ Assmann 1983a, p. 358, parle de Lebensspendenden pour désigner les trois éléments (soleil, Nil et vent).

${ }^{15}$ ERman 1923, p. 154 ; Helck 1970, p. 23 ; Blumenthal 1982, p. 3 ; Dieleman 2003, p. 75 ; QuiRKe 2004, p. 136

${ }^{16}$ Lichtheim 1973, p. 141 ; DeVAuChelle 1994, p. 11 ; PARKINSON 1997b, p. 136.

${ }^{17}$ GARDINER 1914, p. 103 ; SIMPSON 1973, p. 236.

${ }^{18}$ LEFĖBVRe 1949, p. 99 ; POSENER 1956, p. 155.

${ }^{19}$ Foster 2001, p. 79.
} 
Certains commentateurs interprètent ce dérèglement du cycle de la nature comme une référence à un phénomène climatique qui aurait eu lieu durant la Première Période intermédiaire ou à la fin la $\mathrm{XI}^{\mathrm{e}}$ dynastie $^{20}$. D'autres pensent qu'il représenterait de façon imagée les tourments et la confusion en Égypte ${ }^{21}$. En réalité, une autre interprétation est envisageable : ces trois perturbations seraient liées à la séparation du dieu solaire Rê et de l'humanité ${ }^{22}$. Celle-ci est effectivement explicitée plus loin dans le discours du sage et n'est pas sans rappeler le mythe de l'Eil de Rê ${ }^{23}$, tel qu'il est relaté dans le Livre de la Vache du Ciel ${ }^{24}$.

(XI, d-i) Rê se sépare [a] des Égyptiens : il se lèvera quand ce sera l'heure, mais on ne saura plus que midi est arrivé, (car) on ne distinguera plus son ombre, le visage ne sera plus ébloui, quand on (le) regardera, les yeux ne s'embueront plus de larmes, (car) il sera dans le ciel comme la lune [b]. Son moment de la tombée de la nuit ne déviera pas : ses rayons seront assurément dans le visage selon ses dispositions antérieures [c].

[a] En dépit du contexte futur de la prédiction de Néferty, nous avons traduit le verbe îd «séparer» $(W b . \mathrm{I}, 58,12)$ par un présent, car il se trouve à l'aoriste dans le texte. La proposition « Rê se sépare des Égyptiens » est, en effet, sur le même pied que celle « Je te montre (Di.i n.k) le pays dans la détresse » (VIII, e et XII, a), qui introduit les paragraphes relatifs à l'apogée d'Isfet (cf. point 1.1.c). Par conséquent, Néferty annonce le retrait de Rê du monde des humains avec un énoncé à l'aoriste, avant de le détailler dans les phrases suivantes au prospectif.

[b] Comprendre que les rayonnements du soleil seront si faibles qu'ils ressembleront à ceux de la lune.

[c] Posener a suggéré que le pronom suffixe $f$ de $s t w t$ " les rayons » pourrait se référer à ich « la lune $»^{25}$. Le texte soulignerait ainsi l'opposition entre le soleil, qui perdrait son éclat, et la lune, qui conserverait sa luminosité. En effet, $W b$. IV, 331, 14, signale que le terme stwt « les rayons », qui signifie littéralement « projection (lumineuse) », n’est pas réservé au soleil et qu'il pourrait de ce fait se référer à la lune. Ainsi, Posener interprète également le pronom $f$ du substantif $n w$ « le moment» comme désignant l'astre lunaire, mentionné dans la phrase précédente. Alors que Barta a suivi cette interprétation ${ }^{26}$, d'autres traducteurs ont préféré faire

${ }^{20}$ ERMAN 1923, p. 154, n. 3, a proposé d'y voir une description d'une tempête de sable. GoEDICKE 1977, p. 23, qui interprète le texte comme un document historique authentique, y voit un compte-rendu des sécheresses et des famines dont Néferty aurait été témoin. Cf. aussi BELL 1971, p. 17.

${ }^{21}$ GOEDICKE 1977, p. 86, considère la confrontation des vents comme une métaphore des conflits internes entre la lignée héracléopolitaine et la dynastie thébaine durant la $\mathrm{XI}^{\mathrm{e}}$ dynastie.

${ }^{22}$ POSENER 1956, p. 41, avait déjà formulé cette hypothèse pour l'anomalie du soleil. En revanche, il mettait en relation les descriptions de la sécheresse du Nil avec les famines citées dans les textes historiques et biographiques (cf. VANDIER 1936, p. 70-77). En outre, GNIRS 2006, p. 253, souligne également que la désapprobation du dieu concernant les événements terrestres se traduit dans le texte par une perturbation de l'ordre cosmique.

${ }^{23}$ PARKINSON 2002, p. 197, y a déjà discerné l'allusion au mythe de la rébellion et de la destruction de l'humanité. Cependant, il estimait que la Prophétie de Néferty ne discutait pas de l'origine du chaos, car l'intérêt du récit résiderait autour de la venue du roi Amény.

${ }^{24}$ Le récit du Livre de la Vache du Ciel relate la rébellion de l'humanité ainsi que ses conséquences : lorsque les dieux et les humains cohabitaient sur terre sous la tutelle du dieu Rê, ces derniers fomentèrent un complot contre lui à cause de sa sénescence. Rê décida d'envoyer son CEil divin punir les hommes et il se résolut à se séparer du monde terrestre. Voir HORNUNG 1982 ; GUILHOU 1989 ; GUILHOU 1998, p. 197-213.

25 POSENER 1956, p. 155-156.

${ }^{26}$ BARTA 1971, p. 43, n. 24. 
renvoyer les pronoms possessifs de stwt.f «ses rayons » et de $n w . f$ «son moment» au dieu solaire Re $\hat{e}^{27}$. L'ensemble du paragraphe pourrait alors décrire l'éclat du soleil au cours de ses trois phases : le lever du soleil (wbn «briller (à l'aube) ${ }^{28}$ ); le soleil au zénith (mtrt « midi »); le coucher du soleil ( $n w . f n(y)<w>\check{s} s \grave{t}$ « son moment de la tombée de la nuit »). Le texte suggérerait que les rayons du soleil levant et couchant produisent exactement la même luminosité que jadis. Par contre, au milieu de sa course, ses rayons, censés être intenses, seront faibles. C'est la raison pour laquelle le texte établit une comparaison avec la lune : dorénavant, tout au long des trois phases de son cycle, l'astre solaire produira une pâle luminosité constante, à l'image de celle de l'astre lunaire.

En rapport avec cette séparation de Rê, Néferty conclut ses plaintes sur le constat de la déchéance théologique d'Héliopolis, ville du dieu Rê. Cette déclaration constitue l'acmé de la lamentation, puisqu'on y apprend que l'Égypte est abandonnée de tous les dieux ${ }^{29}$.

(XII, g) Le nome héliopolite ne sera plus la terre de naissance d'aucun dieu.

Ces passages nous amènent à interpréter la description de l'obscurcissement du soleil comme une allusion à l'éloignement du dieu solaire Rê. Concernant la sécheresse du Nil et la confrontation des vents, il est possible de saisir leur valeur symbolique en se référant aux sources textuelles décrivant la création du Nil et des vents par le démiurge. Un des textes les plus significatifs à ce sujet est la formule 1130 des Textes des Cercueils ${ }^{30}$, qui constitue la conclusion du Livre des Deux Chemins, daté de la fin de la $\mathrm{XI}^{\mathrm{e}}$ dynastie. Celle-ci contient un monologue du dieu Rê-Atoum, dans lequel il détaille les quatre actions bénéfiques effectuées pour l'humanité, lors de la création de l'univers.

(CT VII 461, c - 464, c) Paroles dites par Celui-dont-les-noms-sont-cachés, le Maître de l'Univers (...) : "Avancez donc en paix, que je vous répète les quatre bonnes actions que mon cour avait conçues pour moi à l'intérieur du Méhen afin de neutraliser le mal (isft). J'ai fait quatre bonnes actions dans le porche de l'horizon. J'ai créé les quatre vents, afin que chacun puisse respirer en son temps; ce fut l'une des actions. J'ai créé la grande inondation, afin que le petit comme le grand soient vigoureux; ce fut l'une des actions. J'ai créé chaque homme semblablement. Je ne les ai pas autorisés à commettre le mal (isft), (mais) leurs cours ont désobéi à ce que j'avais dit; ce fut l'une des actions. J'ai fait que leurs cours s'abstiennent d'oublier l'Occident, afin qu'ils fassent des offrandes divines aux dieux des nomes ; ce fut l'une des actions. »

En somme, Néferty annonce que l'éloignement du dieu Rê a ébranlé deux des quatre bonnes actions qu'il avait réalisées pour l'humanité lors de la création : la sécheresse du Nil prive les Égyptiens d'eau et de nourriture, et l'affrontement des vents rend l'air irrespirable. Des bouleversements atmosphériques similaires, également suscités par le dieu créateur, sont attestés au Nouvel Empire dans des textes de piété personnelle. Dans une supplique au dieu Amon lors d'une année de troubles : "Viens à moi, Amon, et sauve-moi de cette année misérable. Il est arrivé que le soleil ne se lève plus, que l'hiver survienne en été, que les mois arrivent à l'envers et que les heures soient confondues »(P. Anastasi IV, 10, 1-5) ${ }^{31}$. Dans un

\footnotetext{
${ }^{27}$ ERMAN 1923, p. 156 ; LefÈBVRE 1949, p. 103 ; LiCHTHEIM 1973, p. 145, n. 13 ; SiMPSON 1973, p. 238,

${ }^{28}$ Cf. LACAU 1971, p. 1-9.

${ }^{29}$ Cf. PARKINSON 2002, p. 197.

${ }^{30}$ CT VII, 461 c - 471g. Traductions : BARGUET 1986, p. 662-664 ; PARKINSON 1991, p. 32-34.

${ }^{31}$ CAMINOS 1954, p. 170-171.
} n. 17. 
passage relatif à la punition infligée par le dieu à un homme qui a commis une transgres$\operatorname{sion}^{32}$ : «Ton homme violent (litt. chaud) est comme la colère ( $b$ ‘w) d'Amon. Il est l'abomination des hommes. Le soleil ne se lève plus en sa présence, et l'inondation ne coule plus pour lui » (P. Anastasi V, 7, 5-8 = pChester Beatty V, recto 6,7 sq).

En conclusion, les trois perturbations atmosphériques décrites par Néferty auraient pour unique cause l'éloignement du dieu Rê. Une telle conception théologique n'est pas attestée avant le Nouvel Empire. C'est la raison pour laquelle les commentateurs qui situaient la composition de la Prophétie de Néferty au Moyen Empire ne pouvaient concevoir cette résolution divine comme étant à l'origine d'Isfet, mais l'interprétaient comme un renversement théologique résultant des bouleversements climatiques décrits ${ }^{33}$. Néanmoins, comme nous l'avons déjà signalé, les recherches égyptologiques les plus récentes font du début de la $\mathrm{XVIII}^{\mathrm{e}}$ dynastie le terminus ante quem pour la composition du récit de la Prophétie de Néferty. Par conséquent, celui-ci pourrait véhiculer les prémisses d'une conception théologique concernant la séparation du dieu Rê de l'humanité, qui sera développée dans le courant du Nouvel Empire avec le texte cosmogonique qu'est le Livre de la Vache du Ciel.

\section{Développement : l'invasion étrangère}

Le discours de Néferty se poursuit avec l'invasion des étrangers dans le Delta oriental ${ }^{34}$. Il s'agit d'un thème fondamental dans le récit, puisqu'il fut annoncé par le narrateur, avant que le sage n'entame son monologue.

(III, a-e) Il considérait ce qui arriverait dans le pays et il mentionnait la situation de la région orientale, quand les Âmou se déplaceraient avec leurs cimeterres, en terrorisant les cours de ceux qui sont à la moisson et en capturant les attelages en train de labourer.

Néferty commence la description de cette intrusion avec la métaphore de l'oiseau étranger $^{35}$, avant de la détailler en employant un vocabulaire à connotation militaire. Il fait ensuite référence à l'installation des troupeaux étrangers, qui s'abreuveront aux cours d'eau de l'Égypte. Il termine en soulignant la nature dissimulée de cette intrusion.

(VI, f-g) Un oiseau étranger pondra dans les marais de la Basse-Égypte, après avoir établi un nid à côté des Égyptiens. Les gens le laisseront s'approcher à cause du manque.

(VII, e-i) Les ennemis apparaîtront à l'Est, et les Âamou descendront en Égypte. On manquera d'une place forte, si bien qu'un autre [a] sera à côté, sans que les gardes ne (l')entendent. On dressera une échelle dans la nuit, on entrera dans les places fortes et on chassera le sommeil des yeux, si bien que celui qui dormait (dira) [b]: "Je (me) suis réveillé" [c].

(VIII, a-b) Les troupeaux étrangers s'abreuveront aux cours d'eau de l'Égypte [d]. C'est à défaut de quelqu'un qui les chasse qu'ils se rafraîchiront à leurs rives.

\footnotetext{
${ }^{32} \mathrm{Ce}$ texte renseigne sur la condition de celui qui est sous l'emprise de la colère (baou) d'un dieu (cf. KoENIG 1994, p. 220).

${ }^{33}$ Cf. notamment ASSMANN 1983a, p. 358, n. 63.

${ }^{34} \mathrm{Cf}$. les commentaires de FELBER 2005, p. 67-71.

${ }^{35}$ Selon GNIRS 2006, p. 249, cette métaphore aurait pour objectif de comparer le comportement nomade des migrants de l'Est avec celui des oiseaux migrateurs.
} 
(VIII, c-d) Le pays sera instable, et on ne connaîtra pas l'intrusion qui se produira de manière dissimulée, comme on dit: "Vois, l'ouie est engourdie (litt. sourde), si bien que le silence se trouve devant (soi) »[e].

[a] Le terme $k y$ « autre » peut désigner l'étranger et, en l'occurrence, l'envahisseur.

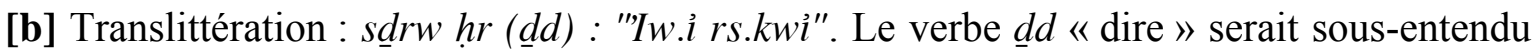
derrière la préposition $h r$ et il introduirait le discours direct ${ }^{36}$. D'autres commentateurs préfèrent supprimer la préposition $h r$, pour faire de $i w . i r s . k w i$ une proposition subordonnée qu'ils traduisent par « (si bien que) je passe la nuit éveillé ${ }^{37}$. Cette lecture doit être écartée, car elle implique que le pronom suffixe à la première personne renverrait à Néferty, ce qui pose un problème d'interprétation. Autres traductions proposées : " le dormeur étant conforme à quelqu'un d'éveillé $»^{38} ;$ " whether I would be sleeping or awake! $»{ }^{39}$.

[c] Comparer avec un passage de l'hymne 1 à Sésostris III : « lui qui épargne à ses serviteurs d'être fatigués et permet aux notables de se reposer jusqu'à l'aube, ses troupes s'occupant de leur tranquillité $(\underline{d} 3 m w . f n k d d w . s n) »^{40}$. La même idée est développée dans la Stèle de la Victoire de Mérenptah : «Les bastions des forteresses sont tranquilles, seul le soleil réveille leurs guetteurs ; les Medjayou sont étendus dans leur sommeil ${ }^{41}$ (KRI IV, 18, 23). Ainsi, on y observe une situation inverse de celle de la Prophétie de Néferty, puisque les troupes et les Medjayou, soldats étrangers au service de l'Égypte, pouvaient se reposer la nuit, faute d'ennemis à repousser.

[d] Ce passage contredit les déclarations de Néferty concernant la sécheresse du Nil (VI, a). Il s'agit en effet d'un monde confus et contradictoire ${ }^{42}$.

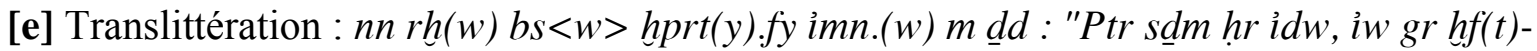
$h r^{\prime \prime}$. De nombreux traducteurs ont compris le complément $m \underline{d} d$ comme introduisant un proverbe, exprimant les effets de l'engourdissement des sens. Dans cette perspective, le terme ptr a été interprété comme le verbe « voir» ou comme la particule « vois », " sache que », attestés dans la langue néo-égyptienne ${ }^{43}$. En nous basant sur le contexte du récit, nous choisissons de comprendre ptr comme une particule proclitique, dans la mesure où la proposition « le silence se trouve devant (soi) » apparaît comme un effet de la diminution de l'audition et non de la vue.

Ces paragraphes fournissent non seulement des informations sur l'invasion proprement dite, mais aussi sur les facteurs qui la favorisent. Néferty reproche d'abord au peuple égyptien de permettre à l'oiseau étranger de s'installer en raison du «manque » $\left(g^{3} w\right)$ qu'il subit. Il

\footnotetext{
${ }^{36}$ Cf. ERMAN 1923, p. 155 ; LefÈBVRE 1949, p. 101 ; PARKINSON 1997b, p. 136 ; DieleMAN 2001, p. 76 ; QUIRKE 2004, p. 137.

${ }^{37}$ GARDINER 1914, p. 105 ; ROEDER 1927, p. 115 ; BARTA 1971, p. 40, n. 13 ; SIMPSON 1973, p. 237.

${ }^{38}$ DeVAuCHelle 1994, p. 12.

${ }^{39}$ FOSTER 2001, p. 80.

${ }^{40}$ GRIFFITH 1898, pl. I, col. 9-10

${ }^{41}$ RosMORDUC 2009, p. 146.

42 PARKINSON 1997b, p. 141, n. 17.

${ }^{43}$ LiChTHEIM 1973, p. 142, «When sight und hearing fail the mute leads »; DeVAUCHELLE 1994, p. 12, « Vois, quand l'entendre est sourd, le silence est présent »; PARKINSON 1997b, p. 136, « See how the hearer is deaf! The mute takes the lead!»; FOSTER 2001, p. 80, « Seeing und hearing shall be blurred and deafened, and silence is in sight»; DiELEMANN 2003, p. 76 : «Zie, wie moet luisteren is doof en de zwijger staat vooraan »; QUIRKE 2004, p. 137, « Look out, the hearer is going deaf, the silent one is before (us) ».
} 
déplore ensuite l'absence $(g$ ว.tw) d'une place suffisamment forte pouvant contrer cette invasion. Simultanément, il blâme les gardes égyptiens qui n'entendent pas ( $n n s \underline{d m}$ ) les ennemis arriver. Dans le troisième paragraphe, les troupeaux étrangers s'abreuvent aux cours d'eau de l'Égypte, à défaut $\left(g^{3} w\right)$ d'un gardien pour les chasser. Finalement, le sage Néferty démontre que cette incursion est favorisée par l'absence de lucidité des Égyptiens. Le proverbe utilisé en guise de conclusion, «Vois, l'ouïe est engourdie (litt. sourde), si bien que le silence se trouve devant (soi) », résume parfaitement l'idée selon laquelle les Égyptiens ont été trompés par leurs sens, en particulier leur ouïe, lors de l'invasion des étrangers.

Nous constatons que, pour justifier la défaite des Égyptiens face à l'ennemi, Néferty recourt systématiquement au substantif $g^{3} w$ 《 le manque » (ou au verbe $g^{3} w$ 《 manquer ») et au verbe $i d i$ « être sourd » (ou au verbe $s \underline{d} m$ « entendre » nié). Il se trouve que les termes $g$ ? $w$ et idi ont été attestés précédemment dans le discours de Néferty, pour exprimer la conséquence majeure de l'obscurcissement du soleil, que nous avons interprété comme une allusion à l'éloignement du dieu Rê : " tout homme sera engourdi (litt. sourd) (id.w) par manque ( $\left.g^{3} w\right)$ de lui (i.e. le soleil) » (V, e). Nous pouvons en déduire que l'absence de Rê aurait plongé les Égyptiens dans un état de torpeur, si bien qu'ils n'ont pas eu connaissance des événements $(n n r h(w) b s<w>$ ), ce qui aurait grandement avantagé les étrangers lors de leur intrusion, que le texte qualifie de « cachée » (imn. $(w))$.

Dans le récit cosmogonique conservé par le texte de la Théologie Memphite ${ }^{44}$, on apprend que l'accès à la connaissance par le dieu Ptah ne peut être rendu possible que par les organes sensoriels : "Les yeux voient, les oreilles entendent et le nez respire. Ils informent le cœur. C'est lui qui produit toute connaissance » (col. 56). Quoique la Prophétie de Néferty insiste sur l'audition engourdie des Égyptiens, les sens visuel et olfactif n'en sont pas moins affectés par les perturbations cosmiques mentionnées supra: l'obscurcissement du soleil diminue la vision, et la confrontation des vents empêche la respiration. Les hymnes solaires illustrent ce principe selon lequel les perceptions sensorielles des Égyptiens sont affinées lorsque le soleil rayonne, mais s'amenuisent dès qu'il se couche : "Tous les yeux voient grâce à toi et ils n'y arrivent plus au moment où Ta Majesté se couche » (Hymne à Amon-Rê de Souty et Hor, 6$7)^{45}$; «Les yeux brillent, les oreilles sont ouvertes, tous les corps sont vêtus quand il brille » (Hymne à Amon, chapitre 9, II, 4-5) ${ }^{46}$; «Quand tu te couches dans l'horizon occidental du ciel, ils dorment en état de mort, leurs têtes sont voilées, et leurs nez sont bouchés ${ }^{47}$ jusqu'à ce que tu te lèves à l'aube dans l'horizon oriental du ciel » (Petit hymne à Aton, 4-5) ${ }^{48}$; « Quand tu te couches dans l'horizon occidental, le pays est dans l'obscurité en état de mort, on dort dans la chambre, et les têtes sont voilées. Un œil ne peut voir son semblable » (Grand Hymne à Aton, 3$)^{49}$.

\footnotetext{
${ }^{44}$ Édition : BREASTED 1901, pl. I-II.

${ }^{45}$ Stèle du British Museum 826, datée du règne d'Aménophis III (édition : VARILLE 1942).

${ }^{46}$ Papyrus Leyde I-350, daté de la fin du règne de Ramsès II (cf. GARDINER 1905).

${ }^{47} \mathrm{~L}$ 'expression « les nez sont bouchés » indique que la respiration est bloquée.

${ }^{48}$ Éditions : DAVIES 1906, pl. XXXII-XXXIII (traduction et commentaires, p. 27-29) ; SANDMAN 1938, p. 10-15.

${ }^{49}$ Éditions : Davies 1908, pl. XXVII (photographies pl. XLI ; description, traduction et commentaires, p. 18-19 et p. 29-31) ; SANDMAN 1938, p. 93-96.
} 


\section{Apogée : le pays dans la détresse}

Les derniers paragraphes des «lamentations » du sage sont introduits par la phrase «le pays sera dans la détresse (sny-mny) ». Son dessein est d'offrir à présent un croquis du pays plongé dans le chaos. On y trouve dès lors divers topoï et procédés stylistiques bien attestés dans les lamentations littéraires, comme la guerre civile, la famine, le vol, l'homicide, les formulations einst-jetzt, etc.

Dans un premier temps, Néferty aborde la décadence du pays en trois paragraphes. Il commence en soulignant la confusion qui y règnera, avec l'éclatement d'une guerre civile et la violence dont feront preuve les Égyptiens pour subvenir à leurs besoins. Par ailleurs, les rites funéraires ne seront plus accomplis, et les homicides deviendront fréquents, de même que les conflits au sein de la cellule familiale.

(VIII, f - IX, f) On prendra les armes de guerre, si bien que le pays vivra dans la confusion. On fabriquera des flèches en cuivre, on cherchera à obtenir du pain par le sang et on rira aux éclats au sujet de la souffrance. On ne pleurera plus à cause de la mort et l'on ne dormira plus affamé à cause de la mort [a], (car) le cour de l'homme s'est tourné vers luimême. On n'accomplira plus les rituels de deuil désormais, (car) le cour se trouve totalement détourné au sujet de ces choses : alors qu'un homme est assis en fermant les yeux [b], un autre est en train de massacrer son prochain. Je te montre un fils comme ennemi, un frère comme adversaire, un homme massacrant son père.

[a] En Égypte ancienne, le jeûne est attesté dans le cadre d'une veillée funèbre ${ }^{50}$.

[b] Translittération : hms $s$, ỉw $k^{c} h . f s^{3} . f, k y h r s m \supset k y$. Gardiner et Lefèbvre se réfèrent à la source du pErmitage, qui note la préposition $r$ devant $k^{c} h . f$, si bien qu'ils traduisent « dans son coin $»^{51}$. Par ailleurs, Lefèbvre a restauré ỉ $f m$ devant $s$. $f$ afin d'avoir un parallèle avec le passage IX, c « le cœur de l'homme s'est tourné vers lui-même $\left(m s^{3} . f \underline{d} s . f\right) »^{52}$. Quant à Posener, il se réfère à l'ostracon GČ 106,2, optant ainsi pour la lecture de l'auxiliaire $i w$, à la place de la préposition $r:$ « un homme se tiendra coi et courbera l'échine $»^{53}$. Enfin, Barta propose la translittération ir et traduit la proposition par « Ein Mann sitzt da; indem er seinen Rücken wendet $»^{54}$. Cependant, comme le terme $s$, littéralement « le dos », peut signifier « la paupière $\rangle^{55}$, c'est-à-dire le dos de l'œil, nous pourrions traduire l'expression $k^{c} h s^{3} . f$ par « fermer les yeux ».

En déplorant, d'une part, l'attitude du peuple égyptien qui agit selon Isfet en commettant des meurtres et, d'autre part, l'inobservance des coutumes funéraires, le texte témoigne de la disparition des troisième et quatrième bonnes actions du dieu solaire, mentionnées dans la formule 1130 des Textes des Cercueils, relatives à l'interdiction de commettre le mal et à la pratique obligatoire des rituels funéraires: "J'ai créé chaque homme semblablement. Je ne les ai pas autorisés à commettre le mal (isft), (mais) leurs cœurs ont désobéi à ce que j'avais dit ; ce fut l'une des actions. J'ai fait que leurs cœurs s'abstiennent d'oublier l'Occident, afin

\footnotetext{
${ }^{50}$ Cf. POSENER 1956, p. 151-152.

${ }^{51}$ GARDINER 1914, p. 104 ; LEFÈBVRE 1949, p. 102.

52 LeFÈBVRE 1949, p. 102.

53 Posener 1956, p. 152-153.

${ }^{54}$ BARTA 1971, p. 41, n. 19.

${ }^{55}$ Cf. BonnAmy et SADEK 2010, p. 657.
} 
qu'ils fassent des offrandes divines aux dieux des nomes ; ce fut l'une des actions » (CT VII, $463, \mathrm{f}-$ VII 464, f).

La description de la décadence du pays se poursuit avec la mention de l'effondrement de la société égyptienne. En effet, Néferty prédit avec amertume que «la création sera comme si elle n'avait jamais existé ». En vue de démontrer ses dires, il fournit des exemples d'inversions de droits de propriété.

(X, b-g) Le pays sera ruiné, (car) on instaurera des lois contre lui qui détruiront ce qui fut fait et qui dévasteront ce qui fut trouvé. La création sera comme si elle n'avait jamais existé. On emportera le bien d'un homme contre son gré, en (le) donnant à celui qui est étranger. Je te montre un propriétaire dans le chagrin et un étranger satisfait.

Le troisième paragraphe nous renseigne sur l'amoindrissement et la dévastation du pays, qui constituent des effets de la prolifération des dirigeants et des taxes.

(XI, b-c) Le pays sera amoindri, mais ses dirigeants seront nombreux. Il sera dévasté, mais les taxes (qui le frapperont) seront considérables. Le grain sera rare, mais le boisseau [a] sera grand: on le mesurera en le faisant déborder.

[a] Le terme ipt désigne une mesure de grains ainsi qu'un récipient de forme cylindrique. À partir de la XVIII ${ }^{\mathrm{e}}$ dynastie, la valeur de cette mesure correspond à 4 heqat (soit 19,076 $\left.\mathrm{dm}^{3}\right)^{56}$.

Dans un second temps, Néferty intensifie sa description chaotique du pays en recourant au topos du monde inversé. Il s'agit du constat qu'à un moment donné, l'ordre de la société est en quelque sorte inversé, c'est-à-dire que les hommes sont dans des situations inverses de celles où ils se trouvaient précédemment. Une des caractéristiques de ce topos est la formulation einst-jetzt (ou en anglais then-now). Attestée dès l'Ancien Empire, elle se présente comme une structure rhétorique et antithétique qui, dans les lamentations littéraires, permet d'appuyer le contraste entre deux périodes, l'une régie par la Maât, l'autre anéantie par Isfet ${ }^{57}$.

(XII, b-c) Celui dont le bras était faible est (maintenant) un maître d'action. On saluera celui qui (autrefois) saluait. Je te montre un subordonné devenu un chef. On suivra celui qui (autrefois) fuyait (?).

Par ailleurs, Westendorf a démontré que le mouvement binaire einst-jetzt se transforme aisément en un mouvement ternaire par le retour à la première situation au terme de la deuxième phase ${ }^{58}$. On discerne une allusion à cette logique cyclique (création-destruction(re)création) dans le souhait formulé par Néferty au début de son discours : "La création est comme si elle n'avait jamais existé. Que Rê (re)commence à fonder le pays » (IV, c-d). Cette vision ternaire sera confirmée dans la suite du récit, avec l'annonce de la venue du roi sauveur Amény, qui restaurera la Maât.

\section{Synthèse}

La distinction entre les causes et les effets des troubles consignés dans la Prophétie de Néferty est pertinente pour mieux définir les caractéristiques littéraires inhérentes au concept d'Isfet. Il était de coutume de considérer que le dérèglement du cycle de la nature et la menace

\footnotetext{
${ }^{56}$ MiCHEL 2014, p. 532.

${ }^{57}$ HeRRMANN 1957, p. 12-15 et 95 ; SEIBERT 1967, p. 20-25 ; SCHENKEL 1984, p. 51-61.

${ }^{58}$ WESTENDORF 1986a, p. 5-8.
} 
étrangère constituaient des caractéristiques typiques du monde égyptien privé de Maât ou que l'ensemble des maux décrits devait être imputé à l'intrusion étrangère. Au terme de l'analyse qui vient d'être proposée, il semble que l'auteur de la Prophétie de Néferty a plutôt voulu présenter celle-ci comme le résultat de l'insouciance des Égyptiens après l'éloignement du dieu solaire vers le ciel. C'est la raison pour laquelle il conviendrait d'interpréter cette intrusion étrangère comme une étape menant à la décadence de l'Égypte, que Néferty expose tout au long des paragraphes relatifs à l'apogée d'Isfet.

\begin{tabular}{|l|l|l|l|}
\hline & Prémices & Développement & Apogée d'Isfet \\
\hline Lamentations & Séparation de Rê & Incursion étrangère & Pays dans la détresse \\
\hline
\end{tabular}

Tableau 1 : Le concept d'Isfet dans les « lamentations » de Néferty

\section{Le roi Amény}

La prédiction de Néferty annonce un dénouement salvateur : un roi nommé Amény ramènera l'ordre (Mâ̂t) et la prospérité dans le pays (XIII, a - XV, f). Cette section commence avec une brève présentation d'Amény, qui offre des analogies avec celle de Néferty au début du récit.

(II, r - III, e) C'était un savant de la région orientale. Il appartenait à Bastet, quand elle brillait. C'était un enfant du nome héliopolite. Il considérait ('Iw.f mh.f) ce qui arriverait dans le pays et il mentionnait (iw.f sḩ.f) la situation de la région orientale, quand les Âmou se déplaceraient avec leurs cimeterres, en terrorisant les cours de ceux qui sont à la moisson et en capturant les attelages en train de labourer.

(XIII, a-f) C'est un roi du Sud qui viendra, dont le nom est Amény, juste de voix. C'est le fils d'une femme du nome de Ta-Séty. C'est un enfant de Khen-Nekhen. Il prendra (Iw.f $r$ šsp) la Couronne Blanche, il portera (iw ff $r$ wts) la Couronne Rouge et il unira (iw.f $r$ sm引) les deux Puissantes. Il satisfera (Iw.f r shtp) les deux Maîtres [a] grâce à ce qu'ils aiment : faire le tour du champ en saisissant la rame et en faisant une onction [...][b].

[a] C'est-à-dire Horus et Seth" .

[b] Translittération : phl $r$ ihy $m h f^{\leftarrow} w s r, m n w d t$ [...]. Barta traduit « Der Acker wird umlaufen beim Fassen des Ruders und beim Bewegen [...] », en proposant de restituer dans la lacune le substantif $r d w y$ «les pieds», afin d'avoir un parallèle avec le terme $w s r$ «la rame $»^{60}$. Bien que Barta opte pour la lecture $n w d$ « bewegen », il suggère que ce terme pourrait être associé au bois- $n w t$ ( $W b$. II, 217, 10), qui est attesté dans Ipou-our, 3.11. Parkinson et Quirke traduisent $m n w d$ par $~ «$ in motion ${ }^{61}$ et $«$ in the jump $»^{62}$, tandis que Simpson propose «swinging ${ }^{63}$. Seul Foster comprend $n w d t$ «swaddling clothes ${ }^{64}$. Néanmoins, dans le

\footnotetext{
${ }^{59}$ Cf. POSENER 1956, p. 52.

${ }^{60}$ BARTA 1971, p. 44, n. 2.

${ }^{61}$ PARKINSON 1997b, p. 138.

${ }^{62}$ QUIRKE 2004, p. 138.

${ }^{63}$ SiMPSON 1973, p. 239.

${ }^{64}$ FosTer 2001, p. 84.
} 
contexte d'un rituel de couronnement royal, nous pourrions lire le terme $n w d t$ «l'onguent » ( $\mathrm{Wb}$. II, 226, 6).

Les deux présentations fournissent d'abord des informations sur l'origine des protagonistes : trois éléments en parallèle font contraster l'origine septentrionale de Néferty (« région orientale », «Bastet» et «nome héliopolite ») et l'origine méridionale d'Amény («Sud », «nome de Ta-Séty» et "Khen-Nekhen ») ${ }^{65}$. Ce procédé stylistique est connu, il s'agit du "parallélisme d'identité », qui consiste à développer une idée - ici les attaches locales d'un personnage -, en la formulant de différentes façons. Ensuite, des propositions introduites par $i w . f$ mettent en parallèle les tâches et les fonctions respectives du ritualiste Néferty et du roi Amény : d'un côté, le devoir de Néferty, qui est de mentionner la situation dans le pays et tout particulièrement celle de la région orientale lors de l'invasion des ennemis; de l'autre, la fonction future d'Amény qui, coiffé de la Double Couronne, règnera conformément aux normes traditionnelles et officielles, en accomplissant les rituels religieux.

L'usage du diminutif 'Imny « Amény » au lieu du nom complet Amenemhat est attesté pour désigner des particuliers de la XII ${ }^{\mathrm{e}}$ dynastie ${ }^{66}$, ainsi que le roi Amenemhat II $^{67}$. Pour Posener, cet hypocoristique a pu également s'appliquer à Amenemhat $\mathrm{I}^{\mathrm{er}}$, comme semble le confirmer un ostracon du règne de Touthmosis $\mathrm{I}^{\mathrm{er}}$ préservant le début de l'Enseignement d'Amenemhat, où le copiste a d'abord écrit le nom 'Imny avant de le corriger en 'Imn- $m$ - $h 3 t$ « Amenemhat ${ }^{68}$.

Le choix de l'auteur de la Prophétie de Néferty d'utiliser le surnom Amény continue de faire l'objet de diverses hypothèses et spéculations. Assmann a proposé d'y voir une référence au roi Ménes $(M n y)$, fondateur de la première dynastie ${ }^{69}$. D'autres analysent le nom Amény comme un nisbé, " celui qui appartient à Amon », qui serait formé à partir du substantif 'Imn "Amon $»^{70}$. Mais il peut également être compris par référence au verbe imn « (être) caché », si bien qu'il signifierait «celui qui est caché » ${ }^{71}$. Cette hypothèse est séduisante, dans la mesure où le roi serait ainsi associé au dieu créateur ${ }^{72}$, qui est décrit dans certains textes comme étant " caché $~^{73}$ : «Paroles dites par Celui-dont-les-noms-sont-cachés (...), le Maître de l'Univers » (CT VII 461, c-d) ; "Si les générations d'hommes passent, c'est depuis que le dieu, qui connaît les caractères, s'est caché » (Mérykarê P 43). Nous pouvons en déduire que l'annonce de la venue d'Amény par le sage Néferty marquerait symboliquement le retour du

${ }^{65}$ PoSENER 1956, p. 35 et 48.

${ }^{66}$ C'est le cas notamment du nomarque de Béni Hassan contemporain de Sésostris $\mathrm{I}^{\text {er }}$ (tombe $\mathrm{n}^{\circ} 2$ ) : NEWBERRY 1893, p. 9 et pl. VII.

${ }^{67}$ Cf. VON BECKERATH 1984, p. 85.

${ }^{68}$ POSENER 1956, p. 23.

${ }^{69}$ ASSMANN 1991, p. 275. Cette hypothèse fut reprise par FrANKE 1994, p. 8 ; GIEWEKEMEYER 2013, p. 346 ; PÉREZ-ACCINO 2015, p. 1498-1499.

${ }^{70}$ STRUVE 1925, p. 220-226 ; LANCZKOWSKI 1960, p. 98, qui estime que l'annonce de la venue d'Amény est liée à une réforme religieuse sous la XII ${ }^{\mathrm{e}}$ dynastie, avec comme dieu principal Amon. FóTI 1976, p. 14, qui suggère qu'au Nouvel Empire, à Thèbes, le récit de la Prophétie de Néferty aurait été interprété comme une description d'Amon avançant triomphalement vers le Nord.

${ }^{71}$ GOEDICKE 1977, p. 14 ; PARKINSON 2002, p. 197 ; GNIRS 2006, p. 252-253.

72 GIEWEKEMEYER 2013, p. 346, propose également d'y voir une allusion au verbe imn « créer », de sorte que le nom Amény pourrait se traduire par « le Créateur».

${ }^{73}$ En raison de sa séparation avec le monde terrestre, il ne peut plus être vu par les humains (cf. PARKINSON 2002, p. 197-198). 
dieu Rê sur terre, dont l'éloignement avait été détaillé dans la section précédente de son discours.

Le texte se poursuit avec la description des initiatives d'Amény pour restituer l'ordre dans le pays, laquelle se développe selon un canevas tripartite qui rappelle la structure des « lamentations ». Mais alors que celle-ci correspondait aux différentes étapes (origine ; développement ; apogée) de la propagation d'Isfet en Égypte, la section consacrée à Amény relate la restauration de la Maât. Nous analyserons et comparerons les thèmes communs des deux sections du discours de Néferty : la création, la menace étrangère et l'ordre du pays.

\section{La création}

Dans l'introduction de son discours, Néferty prévenait le roi Snéfrou qu'il serait amené « à pleurer (rmi) le pays » (III, f), car toute la création serait vouée à la destruction. À l'inverse, il prédit que les gens qui vivront sous le règne d'Amény seront heureux $(r \check{s} w)$. Bien que le sage ait formulé auparavant le souhait que le dieu Rê entame la recréation de l'Égypte, ce sera en définitive Amény qui entreprendra cette tâche, établissant ainsi « son renom éternellement et à jamais $»$.

(IV, c-e) La création sera comme si elle n'avait jamais existé. Que Rê (re)commence à fonder le pays détruit dans sa totalité, (car) il ne restera plus rien : de ce qu'il avait instauré, il n'y aura (même) plus le noir de l'ongle.

(XIV, a-b) Les gens de son entourage se réjouiront, (car) le fils d'un homme [a] établira son renom éternellement et à jamais; $(\mathrm{XV}, \mathrm{f})$ Celui qui verra et sera à la suite du roi sera heureux.

[a] Il s'agit d'une expression qui peut désigner le fils d'un homme illustre. Dans le contexte du récit, elle permet de mettre en exergue l'origine non royale d'Amény ${ }^{74}$.

\section{La menace étrangère}

Avant de pouvoir jouir de cette renommée, Amény livrera combat contre les nomades qui s'étaient installés dans le Delta oriental. Il parviendra à les refouler non seulement grâce aux forces militaires, mais surtout grâce à l'assistance des dieux, qui lui auront transmis les prérogatives de la royauté, à savoir $s n \underline{d}$ « la peur », šct « le massacre », nsrt « la flamme », $\underline{d} n \underline{d} n$ « le courroux » et $\check{s} f \breve{f} f$ « la crainte ${ }^{75}$.

(XIV, c-h) Les malfaiteurs et les rebelles auront abandonné leurs paroles à cause de la peur qu'il (1.e. Amény) inspirera : les Âmou tomberont à cause du massacre qu'il infligera, et les Tjéméhou, à cause de la flamme qu'il allumera, alors que les rebelles seront soumis à son courroux, et les dissidents, à la crainte qu'il inspirera, et l'uraeus qui est à son front pacifiera pour lui les dissidents.

Après sa victoire, Amény construira les «Murs du Souverain », une forteresse défensive destinée à empêcher toute autre incursion des $\hat{A}_{a m o u}{ }^{76}$. Cet ouvrage offre désormais au peu-

\footnotetext{
${ }^{74}$ Cf. Posener 1956, p. 49-50.

75 Blumenthal 1982, p. 26.

${ }^{76}$ Les « Murs du Souverain » ('Inbw H $k_{3}^{3}$ ) sont mentionnés dans Sinouhé (R 43) comme une forteresse « construite pour refouler les Sététyou et pour écraser ceux qui traversent les sables ». Cf. PoSENER 1956, p. 55-57 ;
} 
ple égyptien une protection qui jadis leur a fait défaut (VII, e). Enfin, il s'engagera à contrôler les activités de ces Asiatiques, puisqu'il ne les laissera plus abreuver leurs troupeaux aux cours d'eau de l'Égypte comme bon leur semble.

(XV, a-d) On construira les Murs du Souverain - vivant (soit-il), prospère et en bonne santé ! -, afin qu'il soit interdit aux Âamou de descendre en Égypte. C'est dans l'attitude de suppliants qu'ils chercheront à obtenir de l'eau pour faire boire leurs troupeaux.

\section{L'ordre du pays}

Enfin, Néferty prédit le retour de la Maât sous le règne du roi Amény. Cette déclaration répond brièvement aux paragraphes des lamentations relatifs au "pays dans la détresse », qui décrivaient le monde en proie à Isfet (VIII, f - XII, g). Le concept de Maât, qui recouvre les notions de vérité, de justice et de droit, exprime avant tout, dans ce contexte, l'idée de l'ordre et il s'oppose à Isfet qui est ici le désordre, le chaos ${ }^{77}$.

(XV, e) La Maât reviendra à sa place, alors qu'Isfet sera rejetée à l'extérieur.

Le roi va ainsi inaugurer une nouvelle ère, à l'instar du dieu créateur Rê-Atoum. La même idée est exprimée dans l'inscription biographique de Khnoumhotep II, nomarque de Béni Hassan au milieu de la XII ${ }^{\mathrm{e}}$ dynastie, lorsqu'il affirme, à propos d'Amenemhat ${ }^{\mathrm{er}}$, qu' « il a chassé Isfet, étant apparu en tant qu'Atoum en personne »(Urk. VII, 27, 9-10) ${ }^{78}$.

\section{Synthèse}

Les deux principales sections du discours de Néferty, à savoir les «lamentations » et le " roi Amény ", sont clairement distinctes, dans la mesure où la première concerne le déclin de l'Égypte, tandis que la seconde relate son redressement. Mais il existe des correspondances entre l'énumération des plaintes et l'annonce du salut de l'Égypte. En effet, les trois grandes thématiques (dieux, étrangers et ordre du pays) s'y succèdent dans un ordre identique : la reconnaissance des dieux envers Amény, qui procède aux rituels religieux, conformément à son devoir de roi, contraste avec le détournement de Rê vis-à-vis de l'humanité, déploré par le sage Néferty ; ensuite, Amény refoulera les étrangers dont l'infiltration dans le Delta avait été amèrement relatée par Néferty ; enfin, le roi restaurera la Maât en repoussant Isfet, cataclysme social minutieusement illustré dans les « lamentations ».

Avec cette analyse comparative, différentielle, entre la prédominance d'Isfet d'une part et la restauration de la Maât d'autre part, nous sommes en mesure de justifier la prévalence d'Isfet dans le récit de la Prophétie de Néferty, dans la mesure où elle dépendrait de l'éloignement du démiurge Rê. Rappelons que le sage passe sous silence la raison pour laquelle le dieu solaire a décidé de se séparer de l'humanité, amorçant ainsi la perte de la Maât en Égypte. En revanche, il fournit le contexte dans lequel sa restauration sera opérée : les dieux seront satisfaits des rituels religieux accomplis par le roi Amény. Ainsi compris, ce passage laisse entendre que le dieu Rê aurait abandonné les êtres humains à cause d'une éventuelle négligence de leurs devoirs cultuels.

OBSOMER 1995, p. 345-346, qui argumente en faveur d'un terme désignant une forteresse unique. Voir aussi MONNIER 2012, p. 271-272, pour un résumé des autres interprétations proposées pour 'Inbw Hk 3 .

77 POSENER 1956, p. 57-58.

${ }^{78}$ DE BUCK 1946, p. 11 ; PoSENER 1956, p. 58. 


\begin{tabular}{|l|l|l|l|}
\hline & Dieux & Étrangers & Ordre du pays \\
\hline Lamentations & Séparation de Rê & Incursion étrangère & Prédominance d'Isfet \\
\hline Roi Amény & Satisfaction des dieux & Refoulement des étrangers & Restauration de la Maât \\
\hline
\end{tabular}

Tableau 2 : Les concepts d'Isfet et de Maât dans la Prophétie de Néferty

\section{Analyses intertextuelles}

En vue d'étayer notre hypothèse selon laquelle les transgressions cultuelles des hommes seraient à l'origine de l'éloignement du dieu Rê et, par conséquent, de la décadence du pays dans le récit de la Prophétie de Néferty, nous effectuerons des analyses comparatives entre celui-ci et des textes égyptiens qui abordent les questions suivantes : la théodicée, l'apogée d'Isfet en Égypte et la restauration de la Maât après une période de troubles.

\section{Les théodicées en Égypte ancienne}

Une théodicée cherche à expliquer l'existence du mal dans un monde créé par un dieu bienfaisant ${ }^{79}$. Bien qu'il soit souvent associé aux figures de Seth et d'Apophis, le mal sur terre procède également du libre arbitre des humains, dans la mesure où leur comportement peut mener à la rupture de l'équilibre cosmique et social ${ }^{80}$. Les textes mythologiques, théologiques et littéraires du Moyen et du Nouvel Empire qui abordent cette question nous intéressent particulièrement dans le cadre de l'interprétation du récit de la Prophétie de Néferty, étant donné que celui-ci s'abstient de formuler clairement l'implication des hommes dans l'effondrement de la Maât.

\section{Les sources théologiques}

La formule 1130 des Textes des Cercueils, déjà examinée supra, se présente comme une théodicée énoncée à la première personne par le démiurge Rê-Atoum. Le dieu décrit la création de l'univers, en insistant sur le fait qu'elle est bénéfique pour l'humanité. Mais il se défend ensuite d'être responsable d'Isfet sur terre : ce sont les hommes qui, selon lui, ont contrevenu à son interdiction de commettre le mal ${ }^{81}$.

(CT VII $463 \mathrm{f}-464, \mathrm{~b})$ J'ai créé chaque homme semblablement. Je ne les ai pas autorisés à commettre le mal (isft), (mais) leurs cœurs ont désobéi à ce que j'avais dit.

D'autres textes égyptiens suggèrent que les desseins divins propices à l'humanité et conformes à la Maât auraient été transgressés. Ainsi, la Complainte de Khâkhéperrê-séneb ${ }^{82}$ : «Maât est placée à l'extérieur, et Isfet, à l'intérieur du Conseil. Le dessein des dieux est perturbé, tandis que leurs affaires sont négligées, si bien que le pays se trouve dans la détresse » (recto 11). De même, un texte d'eulogie royale de la Deuxième Période inter-

\footnotetext{
${ }^{79}$ WeStENDORF 1986b, col. 473 ; PARKINSON 2002, p. 130-138 ; FRANDSEN 2003, p. 60-61 ; LOPRIENO 2003, p. 27-56.

${ }^{80}$ GUILHOU 1986, p. 361-362 ; BICKEL 1994, p. 225-228.

${ }^{81}$ LORTON 1993, p. 137 ; SitZLeR 1995, p. 14 et 16-17 ; KEMBOLY 2010, p. 165 et 167.

82 Édition : PARKINSON 1997a.
} 
médiaire ${ }^{83}$, dans lequel le roi est prié de sauver l'Égypte de sa situation catastrophique : «Il trouva l'Égypte détruite également (...) ravagée ; ce que les dieux ont ordonné précédemment, la nature mauvaise l'a détruit » (col. 7, 4-6).

Dans le Livre de la Vache du Ciel, cette prédisposition de l'homme au comportement violent et impie se présente comme une insurrection contre le dieu Rê en raison de sa vieillesse ${ }^{84}$. Celle-ci marque la rupture de l'équilibre qui caractérisait l'Âge d'or, durant lequel les hommes et les dieux cohabitaient sur terre. Mais les modalités de cette conspiration demeurent indéfinies, puisque le texte affirme seulement que les humains ont "formé des projets » (Séthy $\mathrm{I}^{\mathrm{er}}$, col. $2 ; 3 ; 9$ ) et «ont comploté » (Séthy $\mathrm{I}^{\mathrm{er}}$, col. 11 et 12). Le mythème de la rébellion contre le dieu créateur, qui sera bien attesté à l'époque tardive ${ }^{85}$, est également exploité dans le chapitre 175 du Livre des Morts ${ }^{86}$, lorsque le dieu Atoum reproche aux Enfants de Nout ${ }^{87}$, désignation collective pour 1'humanité, d'avoir altéré sa création. Leurs actes pernicieux y sont plus formellement énoncés que dans le Livre de la Vache du Ciel.

(Livre des Morts, chapitre 175, 2-5) Ô Thot, que faut-il faire des Enfants de Nout? Ils ont fait la guerre, ils ont suscité des tumultes, ils ont causé du désordre (isft), ils ont fomenté la rébellion, ils ont massacré, ils ont procédé à des emprisonnements. En effet, ils ont abaissé ce qui était grand, dans tout ce que j'ai créée.

Les méfaits des êtres humains envers le démiurge ou sa création servent d'élément déclencheur de la narration, puisqu'ils perturbent la situation présente et amènent le dieu à prendre des mesures radicales. Dans le Livre de la Vache du Ciel, la conspiration des humains provoque leur massacre, perpétré par la déesse Hathor incarnant l'CEil de Rê, ainsi que le départ du dieu solaire vers le ciel. Dans le chapitre 175 du Livre des Morts, le dieu Thot, informé des crimes commis par les Enfants de Nout, conseille au dieu Atoum d' " écourter leurs années » et de « retrancher à leurs mois » (chapitre 175,6). Une telle répression divine est également attestée dans l'hymne au dieu créateur de l'Enseignement pour Mérykarê : «Il (i.e. le dieu) a massacré ses ennemis et a anéanti ses enfants à cause de leur projet de faire une

${ }^{83}$ Édition : PARKINSON 1999, p. 181-190. Cf. aussi ENMARCH 2007, p. 84.

${ }^{84}$ Mythe thanathologique pour l'origine du mal, cf. KÁKOSY 1964, p. 205-216; HoRNUNG 1982, p. 94-95 ; ASSMANN 1990, p. 174-177 ; ASSMANN 2002, p. 386-388.

${ }^{85}$ Dans la cosmogonie de Neith à Esna, gravée sur un des piliers de la salle hypostyle du temple, datant du règne de Trajan (cf. SAUNERON 1962, p. 253-276; SAUNERON 1969, p. 28-34) ; le Livre du Fayoum, qui contient des manuscrits datés de la période ptolémaïque et romaine, relatifs au traité de la topographie cultuelle de la région du Fayoum (cf. BEINLICH 2013-2017) ; une version parallèle est préservée sur le côté gauche de la porte sud du corridor extérieur du temple de Kom Ombo, datant du règne d'Auguste ou de Tibère (cf. DE MORGAN 1894, p. 131, n. 172 ; YOYOTTE 1962, p. 101); le Papyrus Insinger (XX, 16-17), un enseignement écrit en démotique, contient une référence à une rébellion contre le dieu Prê (cf. LEXA 1926 ; LICHTHEIM 1980, p. 184217) ; enfin, le P. Carlsberg 462, constitue une source démotique fragmentaire attestant une rébellion contre le dieu solaire (cf. SMITH 2000, p. 95-112).

${ }^{86}$ De nombreux spécialistes ont étudié les parallèles entre le chapitre 175 du Livre des Morts et le Livre de la Vache du Ciel, cf. GRAPOW 1931, p. 36, n. 1 ; HORNUNG 1979, p. 517 ; HoRNUNG 1982, p. 90-95 ; OCKINGA 1988, p. 518 ; STADLER 2009, p. 375-380 ; TARASENKO 2016a, p. 42-44.

${ }^{87}$ TARASENKO 2016a, p. 35-48, voit un parallèle entre les Enfants de Nout et les Enfants de la Déchéance du chapitre 17 du Livre des Morts (cf. aussi TARASENKO 2016b ; TARASENKO 2017, p. 31-43). On retrouve une référence à la rébellion des Enfants de Nout dans un traité hermétique, qui mentionne une rébellion contre le dieu créateur : celui-ci, avant de la réprimer, consulte le dieu Hermès, homologue grec du dieu Thot (cf. KÁKOSY 1992, p. 258-261).

${ }^{88}$ Édition : NAVILLE 1886, pl. CXCVIII-CXCIX. 
rébellion » $(\mathrm{P} 133-4)$; «Il (i.e. le dieu) a massacré des rebelles parmi eux à la manière dont un homme frappe son fils à cause de son frère » (P 137-8).

\section{Les lamentations d'Ipou-our}

L'analyse des Lamentations d'Ipou-our ${ }^{89}$ est pertinente pour mieux appréhender les aspects de théodicée que la Prophétie de Néferty traite plus tacitement. Dans cette pièce poétique, le sage Ipou-our adresse un discours au Maître de l'Univers, pour se lamenter sur le chaos dans lequel se trouve plongée l'Égypte. Le débat entre Ipou-our, qui apparaît comme le représentant de l'humanité, et le Maître de l'Univers, qui défend les intérêts royaux et divins, porte sur la responsabilité des troubles dans le pays.

Tout au long de ses lamentations, le sage Ipou-our dresse un portrait très négatif de l'humanité. Dans cette perspective, il se réfère au mythème de la rébellion contre le dieu Rê $\hat{e}^{90}$ : "Sachez qu'on en est venu à se rebeller contre l'uraeus [puissant] de Rê $\hat{~}^{91}$ qui pacifie les Deux Terres » (7.3). La complainte se poursuit avec le motif de la destruction des humains rebelles par le dieu créateur. Mais au lieu de le considérer comme un acte répressif justifié, comme dans le Livre de la Vache du Ciel, Ipou-our critique vivement le démiurge, qu'il considère comme négligent ${ }^{92}$. Il lui reproche, en effet, d'avoir épargné l'humanité, alors que « le feu appartenait à son cœur » $(12.2)^{93}$. Dans un passage précédent, Ipou-our formulait déjà le souhait que l'humanité périsse, en raison de sa nature, vile et perfide.

(Ipou-our, 12.2-3) Puisse-t-il s'apercevoir de leur nature à la première génération, il ferait alors opposition, il lèverait le bras contre elle et il détruirait son troupeau et son héritage ;

(Ipou-our, 5.14 - 6.1) Si seulement c'était la fin de l'humanité, sans conception et sans naissance, de sorte que le pays serait silencieux de bruit, sans tumulte.

Ipou-our remet aussi en question le bien-fondé de la création, en reprochant au démiurge son manque de différenciation entre les bons et les mauvais caractères ${ }^{94}$. Cette critique fait allusion à la revendication bienfaisante du dieu Rê-Atoum dans la formule 1130 des Textes des Cercueils, selon laquelle il avait créé chaque homme comme son semblable ${ }^{95}$.

(Ipou-our, 11.12) Voyez, pourquoi cherche-t-il (1.e. Rê) à créer (l'humanité), alors que le peureux n'a pas été distingué du vaillant?

Enfin, une critique sur l'inactivité du dieu Rê est suggérée dans une métaphore nautique négative, dans laquelle le pays est considéré comme un bateau, son gouverneur, comme le

\footnotetext{
${ }^{89}$ Édition : ENMARCH 2008.

${ }^{90}$ Idem, p. 130.

${ }^{91}$ Symbole de l'autorité royale, l'uraeus peut également identifier le dieu créateur dans son combat contre les forces du chaos (SAUNERON 1953, p. 68 et 85), si bien que les rebelles mentionnés feraient référence aux ennemis du démiurge d'un point de vue cosmique ( $W b$. IV, 87, 14).

${ }^{92}$ ENMARCH 2008, p. 57.

${ }^{93}$ L'affirmation selon laquelle le feu appartient au cœur humain constitue une métaphore de la nature destructrice des hommes. Une telle conception était déjà développée dans le passage 11.13, qui associait la chaleur $\left(t^{3} w\right)$ au $s h m-i b$ « celui au cœur violent 》. Cf. ENMARCH 2008, p. 183.

${ }^{94}$ Отто 1951, p. 26.

${ }^{95}$ FeCHT 1972, p. 126 ; ENMARCH 2008, p. 182.
} 
pilote $^{96}$ : «Il n'y a plus de pilote (à bord d'un bateau) en service. Où est-il aujourd'hui ? Estce qu'il dort réellement ? Voyez, sa puissance $(b$ `w) ne peut plus être vue » (12.5).

Dans ses réponses à Ipou-our, le Maître de l'Univers prend la défense des dieux en mettant en exergue leurs souffrances, causées par le comportement impie des hommes, qui saccagent les monuments divins. Cette déclaration de la non-transcendance des dieux vise à les innocenter de l'existence des maux sur terre, en incriminant l'humanité, qui a rejeté les dieux plus qu'ils ne l'ont abandonnée ${ }^{97}$.

(Ipou-our, 16.13-14) Si on les appelle, ils n'écoutent pas [...], les dieux pleurent, car leurs suivants sont entrés dans les chapelles, les statues ont été brûlées, les tombes ont été détruites, ainsi que les corps des momies.

En conclusion, Ipou-our voit dans la nature imparfaite des hommes la source originelle des fléaux qui sévissent sur terre ${ }^{98}$, en lien avec le concept négatif de l'idéologie égyptienne. Mais il impute la situation chaotique présente au dieu Rê qui n'a pas pris les bonnes décisions, que ce soit lors de la création ou lors de l'insurrection de l'humanité. Sa passivité est également vivement reprochée par le sage Ipou-our, qui attend désespérément une manifestation divine. Ce comportement inactif et distant du dieu créateur concorde avec la théologie du Moyen Empire : les textes conservés de cette période attestent rarement une intervention divine en faveur de l'humanité ${ }^{99}$.

\section{L'apogée d'Isfet en Égypte}

D'après les analyses littéraires de la Prophétie de Néferty effectuées supra, l'origine des calamités dans le récit serait à rechercher dans la résolution prise par le dieu Rê de se séparer du monde terrestre, plongeant ainsi la société égyptienne dans Isfet. Considérer le démiurge comme étant à l'origine de la destruction de la création n'est pas nouveau. Il s'agit d'une question eschatologique abordée dans les textes théologiques. Par ailleurs, des sources royales du Nouvel Empire attribuent une prédominance d'Isfet sur terre à une intervention du dieu créateur Amon-Rê en défaveur des humains. Il serait donc pertinent d'analyser la représentation et la conception d'Isfet, aussi bien dans les textes eschatologiques que dans les inscriptions royales du Nouvel Empire, en vue d'une comparaison avec la Prophétie de Néferty.

\section{Les textes eschatologiques}

Dans la Prophétie de Néferty, l'état du pays plongé dans Isfet est défini comme une négation de la création, et Rê est invité à recommencer son œuvre créatrice.

(IV, c-e) La création sera comme si elle n'avait jamais existé. Que Rê (re)commence à fonder le pays détruit dans sa totalité, (car) il ne restera plus rien : de ce qu'il avait instauré, il n'y aura (même) plus le noir de l'ongle; (V, a) Le pays sera détérioré ; (X, d) La création sera comme si elle n'avait jamais existé.

\footnotetext{
${ }^{96}$ ENMARCH 2008, p. 27 et 185.

${ }^{97}$ Idem, p. 57.

98 PARKINSON 2002, p. 209.

${ }^{99}$ BiCKEL 1994, p. 221.
} 
Un parallèle peut être établi avec la littérature théologique égyptienne qui conçoit une fin du monde, annoncée par le créateur, à laquelle seuls lui et Osiris survivront ${ }^{100}$. Un tel discours de nature eschatologique figure dans la formule 1130 des Textes des Cercueils et dans le chapitre 175 du Livre des Morts ${ }^{101}$.

(CT 1130 VII 467e - 468b) J'ai instauré des millions d'années entre moi et cet Immobiledu-cœur, fils de Geb, (puis) je siégerai avec lui dans une seule (et même) place: les buttes deviendront des villes et vice-versa, un temple ruinera un temple [a].

(Livre des Morts, chapitre 175, 17-19) Je (i.e. Atoum) détruirai tout ce que j'ai créé, et ce pays reviendra à l'état de Noun, à l'état de flot, comme son premier état. Je suis celui qui restera avec Osiris, après que je me serai transformé en serpent, que les hommes ne peuvent connaître et que les dieux ne peuvent voir ${ }^{102}$.

[a] Il s'agit du topos du monde inversé. Nous ne retenons pas la traduction de Lichtheim « while hills became towns and towns hills, for dwelling destroys dwelling ${ }^{103}$, qui fut rejetée par Assmann ${ }^{104}$.

C'est sous la forme d'un serpent ${ }^{105}$, symbole de renaissance, qu'Atoum s'assoupira dans les eaux du Noun ramenant ainsi la création à son état initial ${ }^{106}$. Cette conception de la fin du monde est probablement intégrée au récit du Naufragé, où le serpent serait une allusion au démiurge Rê-Atoum ${ }^{107}$ : le discours qu'il prononce au sujet de la destruction de l'île pourrait constituer une métaphore pour celle de l'univers.

\section{Les sources royales du Nouvel Empire}

Au Nouvel Empire, certains discours royaux recourent au concept d'Isfet pour illustrer le chaos dans lequel se serait trouvée plongée l'Égypte avant l'avènement du souverain régnant ${ }^{108}$. L'exemple le plus éloquent est la Stèle de la Restauration de Toutânkhamon ${ }^{109}$, qui, en proposant une description du pays dans la détresse (sny-mnt), rappelle le récit de la Prophétie de Néferty. Mais la stèle de Toutânkhamon est beaucoup plus concise sur le croquis des calamités s'abattant sur l'Égypte, puisqu'elle n'expose que deux fléaux : la défaite militaire et l'inaccessibilité des dieux.

(Urk. IV, 2017, 11-20) Le pays était dans la détresse (sny-mnt), (car) les dieux se détournaient de ce pays. Si l'on envoyait des troupes au Djahy pour étendre les frontières de l'Égypte, elles n'avaient aucun succès. Si l'on priait un dieu pour lui demander quelque

\footnotetext{
100 OTTO 1962, p. 249-256 ; BERGMAN 1983, p. 51-60 ; ASSMANN 1983a, p. 352-353 ; BICKEL 1994, p. 228231 ; LESKO 2006, p. 63-70.

${ }^{101}$ BARGUET 1967, p. 260-263; HORNUNG 1979.

102 Édition : BudGe 1894, pl. XXIX.

${ }^{103}$ LiCHTHEIM 1973, p. 132.

104 ASSMANN 1983a, p. 352, n. 29.

${ }^{105}$ La formule 717 des Textes des Cercueils offrirait une référence au dieu Atoum sous la forme d'un serpent : «Atoum a mordu, il a rempli sa bouche et il s'est enroulé » (CT VI 346 b-c ; BARGUET 1986, p. 608).

${ }^{106}$ KÁKosy 1963, p. 19 ; MeEKS, FAVARD-MeEKS 1995, p. 29-30.

${ }^{107}$ DerChain-UrTel 1974, p. 83-104 ; BAINES 1990, p. 65-67 ; CANNUYER 1998, p. 27-42.

${ }^{108}$ ASSMANN 1983a, p. 364, affirme que les descriptions de troubles politiques dans les sources royales du Nouvel Empire se réfèrent généralement à des périodes de crises authentiques.

${ }^{109}$ Éditions : LACAU 1909, pl. LXX ; Urk. IV, 2025-2032. Voir aussi GABOLDE 2015, p. 124-133.
} 
chose, il ne venait pas du tout. Si l'on priait une déesse pareillement, elle ne venait pas non plus. Leurs cours étaient faibles dans leurs corps, (car) ce qui avait été fait les avait détruits.

Alors que, dans la conception théologique du Moyen Empire, les dieux abandonnaient l'Égypte à son sort quand l'ordre était jeté à bas (cf. les Lamentations d'Ipou-our), inversement, selon la nouvelle conception du Nouvel Empire, c'est parce que les dieux se détournent de l'Égypte que l'ordre est bouleversé ${ }^{110}$. En effet, dans la Stèle de la Restauration, les troubles décrits procèderaient de l'indifférence qu'éprouvent les divinités à l'égard de 1'Égypte ${ }^{111}$. De même, selon la Stèle d'Éléphantine de Sethnakht ${ }^{112}$, c'est au désintérêt des dieux qu'est attribuée la situation déplorable du pays avant l'avènement du roi Sethnakht. Celle-ci est détaillée dans la notice du pHarris $\mathrm{I}^{113}$, qui décrit la période d'anarchie ayant marqué la fin de la XIX ${ }^{\mathrm{e}}$ dynastie.

$(\mathrm{KRI} \mathrm{V}, 672,1)(\ldots)$ depuis que ce pays était dans la désolation, parce que Ta-Méri était tombée dans l'oubli de la divinité.

(pHarris I. 75, 2 - 6) Le pays de Kémet était abandonné (...) : chacun suivait sa (propre) loi, car on n'avait plus de commandant (...). Les dieux étaient traités comme des hommes, car on ne présentait plus d'offrandes à l'intérieur des temples.

Par ailleurs, les événements décrits dans la Stèle de la Restauration sont liés par un rapport de cause à effet. En effet, le texte assigne une causalité au détournement des dieux, à savoir l'abolition des cultes traditionnels durant la période amarnienne. Celle-ci est dénoncée à travers les descriptions du délabrement des monuments divins (temples, chapelles et sanctuaires).

(Urk. IV, 2027, 1 - 10) Or, Sa Majesté est apparue en qualité de roi, alors que les temples des dieux et des déesses depuis Éléphantine [jusqu'aux] marais du Delta [...] tombaient en ruine. Leurs chapelles devenaient délabrées, transformées en décombres envahis de buissons. Leurs sanctuaires étaient comme s'ils n'avaient jamais existé, et leurs domaines étaient des chemins piétonniers.

L'idéologie égyptienne s'exprime rarement explicitement sur les défaveurs des dieux envers les rois ${ }^{114}$. Deux exemples peuvent être cités : un texte de Ramsès IV, qui attribue la faiblesse de l'inondation à l'époque de ses prédécesseurs qui auraient négligé le culte d'Osiris (KRI VI, 21, 7-8), et l'Enseignement pour Mérykarê, dans lequel la faute du roi engendre une punition divine (P 120-122) $)^{115}$.

\section{Le retour à la Maât dans les sources royales du Nouvel Empire}

Le retour de la Maât, tel que décrit dans la Prophétie de Néferty, constitue un trait caractéristique de l'idéologie royale, selon lequel le souverain se doit d'être le garant de l'ordre. De même, les inscriptions royales du Nouvel Empire exprimant Isfet ont pour dessein de glorifier le souverain régnant, dans la mesure où il se vante d'avoir triomphé du chaos, réhabilitant

\footnotetext{
${ }^{110}$ ASSMANN 1983b, p. 285-286, n. 366.

111 VERNUS 1995, p. 143.

112 Édition : KRI V, 671-672.

113 GRANDET 1994, p. 335-336.

114 VERNUS 1995, p. 144-145.

${ }^{115}$ Idem, p. 145.
} 
ainsi la Maât, conformément à son devoir de roi. Il serait intéressant d'examiner de quelle manière les divinités, dont le courroux a fait succomber le pays dans Isfet, interviennent désormais dans la restitution de la Maât et soutiennent le nouveau roi.

Le début de la Stèle de la Restauration met en exergue la restitution des monuments divins et des cultes par Toutânkhamon, grâce à laquelle « le pays fut comme la Première Fois », soit dans le même état que lors de la création ${ }^{116}$.

(Urk. IV, 2016, 16 - 19) Après avoir restauré ce qui était en ruine par des monuments pour l'éternité, il a écarté Isfet à travers les Deux Terres, Maât étant rétablie [à sa place]. Il a fait que l'iniquité (grg) fût en abomination et que le pays fût comme la Première Fois.

Alors que la stèle caractérisait la période amarnienne par un désintérêt des dieux, elle insiste considérablement sur la reconnaissance et la satisfaction de ceux-ci envers le roi Toutânkhamon, pour les mesures et les initiatives qu'il a prises.

(Urk. IV, 2030, 9 - 16) «Je (i.e. Ma Majesté) ferai en sorte qu'ils soient les protégés et les privilégiés auprès de mes pères, tous les dieux, afin de les satisfaire en accomplissant ce que leurs kaou désirent, de sorte qu'ils protègent [l'Égypte]. " Les cœurs des dieux et des déesses qui sont dans ce pays se réjouissaient, et les maîtres des chapelles étaient en joie. Les rives jubilaient, et l'exultation était à travers [le pays, car des décisions] parfaites avaient été prises.

La restauration des monuments ruinés, comme symbole du rétablissement de la Maât, est attestée dans les sources royales dès le début du Moyen Empire, avec l'inscription de Sésostris $\mathrm{I}^{\mathrm{er}}$ à Tôd ${ }^{117}$. Au Nouvel Empire, de nombreux souverains recourent à ce procédé littéraire pour conforter leur légitimité. Ainsi, l'Inscription du couronnement de Horemheb, gravée au dos de la statue Turin $1379^{118}$, utilise une phraséologie similaire à celle de la Stèle de la Restauration: «Or, Elle (i.e. Sa Majesté) (re)fonda ce pays, en l'organisant comme à l'époque de Rê, elle restaura les temples des dieux, depuis les marais du Delta jusqu'à Ta-Séty, et elle façonna toutes leurs images (...). Rê se réjouit en les voyant, elles qui avaient été trouvées ruinées à l'époque précédente» (22-23). Et dans la chapelle rupestre d'Aÿ au nord d'Akhmîm, le début du texte de Nakhmin est également pertinent : " Alors Sa Majesté rechercha ce qui serait utile pour satisfaire toutes les divinités, pour restaurer le(s) sanctuaire(s) des dieux et pour célébrer leurs cultes originels sur terre qui risquaient de s'en aller en décrépitude $\gg(2-3)^{119}$.

Une illustration de retour à l'ordre est en outre attestée dans la Stèle de la Victoire du roi Mérenptah (Caire CG 34025). Celle-ci présente le souverain comme le libérateur de l'Égypte, grâce à sa victoire sur les Libyens en l'an 5 de son règne. Les épithètes royales figurant au début du texte font allusion aux conditions de vie du peuple égyptien, qui avaient été rendues difficiles notamment à cause de perturbations atmosphériques (obscurcissement du soleil et absence d'air), ce qui n'est pas sans rappeler la Prophétie de Néferty (V, c-e et VI, e).

\footnotetext{
${ }^{116}$ Concernant le modèle de la « Première Fois » dans l'idéologie royale, cf. VERNUS 1995, p. 36-38.

${ }^{117}$ Cf. BARbotin, Clère 1991.

118 Cf. GARDINER 1953.

${ }^{119}$ Cf. GabOLDE 2015, p. 457.
} 
(KRI IV, 13, 9-12) Le roi de Haute et de Basse-Égypte Baenrê, aimé d'Amon, le fils de Rê, Mérenptah, (...) le soleil qui dissipe les nuages de tempête qui étaient sur l'Égypte et qui permet à l'Égypte de voir les rayons de l'astre solaire, qui enlève la colline de cuivre du cou de l'humanité, afin de donner le souffle aux gens qui étaient enfermés.

Ces épithètes ont pour dessein de glorifier le roi Mérenptah, dont la venue et les initiatives ont permis un retour à l'ordre cosmique : il a dissipé les nuages dans le ciel et il a donné de l'air au peuple égyptien. En ayant de l'emprise sur les forces cosmiques, le roi est assimilé au dieu créateur et il exprime par conséquent la volonté divine dans sa reprise en main de l'Égypte. Le regain d'intérêt du dieu créateur pour le peuple égyptien est explicitement énoncé dans la suite du texte : "Rê s'est retourné ( $p n^{\prime}$ ) vers l'Égypte » (KRI IV, 18, 15 - 19, 1). Cette expression peut être mise en parallèle avec un passage de la notice du pHarris I, qui décrit l'avènement du roi Sethnakht, fondateur de la $\mathrm{XX}^{\mathrm{e}}$ dynastie $^{120}$.

(pHarris I. 75, 6-7) Mais lorsque les dieux se sont retournés (pn`.w) vers la paix, pour faire que le pays fût (de nouveau) semblable à son état exact, ils ont établi leur fils charnel comme souverain (...) de tout pays à leur grande place, Ouserkhârê-Sétepenrê, aimé d'Amon, (...), le fils de Rê, Sethnakht-Mererrê, aimé d'Amon (...).

En somme, d'après les sources royales du Nouvel Empire analysées, la restauration de l'ordre en Égypte dépend de la volonté des dieux : de la même manière que le courroux des dieux a fait tomber le pays dans Isfet, la Maât ne retournera à sa place qu'une fois que les dieux se soucieront et soutiendront l'Égypte ${ }^{121}$. Vernus établit avec raison un parallèle avec les textes de piété personnelle du Nouvel Empire, qui louent le dieu pour la clémence dont il a fait preuve à l'égard d'un particulier. Celui-ci aurait été accablé d'un malheur (échec professionnel, maladie, infirmité, etc.), suscité par le dieu en colère, et sa guérison ou son succès dépendrait uniquement de la disposition du dieu à lui pardonner son péché ${ }^{122}$.

(KRI VI, 332, 10-12) Voyez, Amon s'est retourné (pn') vers l'Égypte. Celui qui était pauvre est (maintenant) un notable $e^{123}$.

(Stèle Berlin 23077, 8-12) (...) le scribe Nakht-Amon, juste de voix, alors qu'il était couché, malade, aux portes de la mort (...). Le maître était prêt à être miséricordieux. Le maître de Thèbes ne passe un jour entier en colère ${ }^{124}$.

(oCaire 12202, recto, 1-3) Amon-Rê, grand de courroux (b’3), seigneur de miséricorde, tu as fait que je voie le jour comme la nuit. Tu as éclairé (ma) vue (litt. l'œil), t'étant retourné ('n.ti) (vers la clémence) ${ }^{125}$.

${ }^{120}$ Rapprochement déjà effectué par VERNUS 1995, p. 147.

${ }^{121}$ VeRNUS 1995, p. 144, souligne que cette conception idéologique s'est accentuée durant la Basse Époque. En effet, dans le texte démotique La Prophétie de l'Agneau, les malheurs constituent une "malédiction qu'a faite Prê (contre) l'Égypte », Prê étant la forme tardive du nom Rê (cf. RITNER 1993, p. 46 ; DEVAUCHELlE 1994, p. 30).

122 VERNUS 1995, p. 149.

${ }^{123}$ Il s'agit d'un passage issu d'un hymne à Ramsès VI (pTurin 1893 r³, 3). Cf. VERNUs 1995, p. 148

124 Édition : ERMAN 1911, p. 1092-1094.

${ }^{125}$ Édition : POSENER 1975, p. 201-202 et pl. 19. 


\section{Conclusion}

$\mathrm{Au}$ terme de cette analyse comparative, nous sommes en mesure d'émettre une hypothèse argumentée quant à l'origine de l'apogée d'Isfet dans le récit de la Prophétie de Néferty. Celle-ci procèderait de la séparation du dieu Rê et des êtres humains, qui auraient manqué à leurs devoirs cultuels. Dans les textes étudiés, cette impiété se manifeste dans la rébellion mythique de l'humanité contre le dieu créateur (Livre de la Vache du Ciel, Lamentations d'Ipou-our, chapitre 175 du Livre des Morts, Enseignement pour Mérykarê) ou dans la destruction des monuments divins (Lamentations d'Ipou-our, Stèle de la Restauration de Toutânkhamon).

En illustrant l'Égypte en proie à Isfet, le récit de la Prophétie de Néferty offre des descriptions analogues à une fin des temps motivée par le dieu créateur et il constitue à ce titre un parallèle pertinent avec les sources textuelles eschatologiques (formule 1130 des Textes des Cercueils et le chapitre 175 du Livre des Morts). De même, le sage Ipou-our, dans ses Lamentations, en vint à espérer un cataclysme divin, quand il implore le dieu créateur de punir les humains pour leur malfaisance innée. Une telle répression divine est illustrée dans l'Enseignement pour Mérykarê, le chapitre 175 du Livre des Morts ou encore le Livre de la Vache du Ciel. Enfin, les inscriptions royales du Nouvel Empire qui développent le concept d'Isfet se recoupent au sujet de l'origine du chaos sur terre, puisqu'elles l'attribuent à un désintérêt divin (Stèle de la Restauration, Stèle d'Éléphantine de Sethnakht).

Le dieu conserve une maitrise permanente sur sa création, si bien que la restitution de la Maât après une période troublée ne dépendra que de son bon vouloir. Dans la Prophétie de Néferty, la venue du roi Amény symbolise le retour du dieu Rê sur terre, après qu'il s'est séparé des êtres humains. Par ailleurs, les dieux expriment leur satisfaction pour l'accomplissement des cultes qu'il effectue, en lui assurant leur soutien dans son combat contre les Asiatiques installés en Égypte. De la même manière, dans les inscriptions royales du Nouvel Empire, l'emphase est mise sur la reconnaissance des dieux envers le roi qui restitue l'ordre dans le pays (Stèle de la Restauration, Stèle de la Victoire de Mérenptah). Mais, contrairement à la Prophétie de Néferty, elles mentionnent parfois explicitement l'indulgence divine (Stèle de la Victoire de Mérenptah, règne de Sethnakht dans le pHarris I).

Grâce à l'analyse des sources royales qui expriment le concept d'Isfet, il est également possible de mettre en exergue la finalité de celui-ci dans la Prophétie de Néferty : les descriptions des troubles politiques et sociaux ont pour dessein de stigmatiser une période donnée pour mieux glorifier le nouveau souverain Amény. En restaurant la Maât, ce dernier instaure une nouvelle ère et il est dès lors comparé au dieu créateur qui a mis fin au tohu-bohu originel et a organisé l'univers. 


\section{BIBLIOGRAPHIE}

ASSMANN 1983a : J. ASSMANN, « Königsdogma und Heilserwartung: politische und kultische Chaosbeschreibungen in ägyptischen Texten ", dans D. Hellholm (éd.), Apocalypticism in the Mediterranean World and the Near East, Proceedings of the International Colloquium on Apocalypticism. Uppsala, August 12-17, 1979, Tübingen, p. 345-377.

Assmann 1983b: J. Assmann, Re und Amun: Die Krise des polytheistischen Weltbilds im Ägypten der 18.-20. Dynastie (Orbis biblicus et orientalis, 51), Fribourg et Göttingen.

ASSMANN 1990 : J. Assmann, Ma'at: Gerechtigkeit und Unsterblichkeit im Alten Ägypten, Munich.

Assmann 1991 : J. Assmann, Stein und Zeit: Mensch und Gesellschaft im Alten Ägypten, Munich.

ASSMANN 1994 : J. ASSMANN, « Maat und die gespaltene Welt oder: Ägyptertum und Pessimismus », Göttinger Miszellen, 140, p. 93-100.

BAINES 1990 : J. BAINES «Interpreting the Story of the Shipwrecked Sailor », Journal of Egyptian Archaeology, 76, p. 55-72.

BARbotin, Clère 1991 : Ch. BARbotin, J.J. Clère «L'inscription de Sésostris I ${ }^{\text {er }}$ à Tôd », Bulletin de l'Institut français d'archéologie orientale, 91, p. 1-32.

BARguet 1967: P. BARguet, Le Livre des Morts des anciens Égyptiens (Littératures anciennes du Proche-Orient, 1), Paris.

BARguet 1986 : P. BARguet, Les textes des sarcophages égyptiens du Moyen Empire (Littératures anciennes du Proche-Orient, 11), Paris.

BARTA 1971 : W. BARTA, «Zu einigen Textpassagen der Prophezeiung des Neferti », Mitteilungen des deutschen archäologischen Instituts, Abt. Kairo, 27, p. 35-45.

BeINLICH 2013-2017 : H. BeINLICH, Der Mythos in seiner Landschaft: das ägyptische "Buch vom Fayum", 3 vols (Studien zu den Ritualszenen altägyptischer Tempel, 11), Dettelbach.

Bell 1971 : B. Bell, «The Dark Ages in Ancient History I. The First Dark Age in Egypt», American Journal of Archaeology, 75, p. 1-26.

BERGMAN 1983 : J. BERGMAN, «Introductory Remarks on Apocalypticism in Egypt », dans D. Hellholm (éd.), Apocalypticism in the Mediterranean World and the Near East, Proceedings of the International Colloquium on Apocalypticism, Uppsala, August 12-17, 1979, Tübingen, p. 51-60.

BiCKel 1994 : S. BICKEL, La cosmogonie égyptienne. Avant le Nouvel Empire (Orbis biblicus et orientalis, 134), Fribourg et Göttingen.

Blumenthal 1982 : E. Blumenthal, «Die Prophezeiung des Neferti », Zeitschrift für ägyptische Sprache und Altertumskunde, 109, p. 1-27.

BonnAmy et SADEK 2010 : Y. BonnAmy et A.-A. SADEK, Dictionnaire des hiéroglyphes : hiéroglyphes-français, Arles.

Breasted 1901 : J.H. Breasted, « The Philosophy of a Memphite Priest », Zeitschrift für ägyptische Sprache und Altertumskunde, 39, p. 39-54.

Budge 1894 : E.A.W. Budge, The Book of the Dead: Facsimile of the Papyrus of Ani in the British Museum, $2^{\mathrm{e}}$ éd., Londres. 
CAminos 1954 : R.A. CAminos, Late Egyptian Miscellanies (Brown Egyptological Studies, 1), Londres.

CANNuYer 1998: Chr. CANNUYeR, «Le voyage comme tension eschatologique dans l'Égypte ancienne : les leçons du Naufragé », dans Chr. CANNUYER, A. VAN TONGERLOO (éd.), Les voyages dans les civilisations orientales (Acta orientalia belgica, XI), Bruxelles, p. $27-42$.

Davies 1906 : N. de G. Davies, The Rock Tombs of el Amarna, vol. 4. Tombs of Penthu, Mahu, and others, Londres.

Davies 1908 : N. de G. DaVIES, The Rock Tombs of el-Amarna, vol. VI. Tombs of Parennefer, Tutu and $A \ddot{y}$, Londres.

DE BuCK 1946: A. DE BuCK, «La littérature et la politique sous la douzième dynastie égyptienne », dans M. DAVID, B.A. VAn Groningen, E.M. MeIJERS (éd.), Symbolae ad jus et historiam antiquitatis pertinentes Julio Christiano van Oven dedicatae (Symbolae van Oven), Leyde, p. 1-28.

De Morgan 1894 : J. De Morgan, U. Bouriant, G. Legrain (et alii), Catalogue des monuments et inscriptions de l'Égypte antique, t.1. De la frontière de Nubie à Kom Ombos, Vienne.

Derchain-Urtel 1974 : M.T. Derchain-Urtel, « Die Schlange des "Schiffbrüchigen" », Studien zur altägyptischen Kultur, 1, p. 83-104.

Devauchelle 1994 : D. Devauchelle, «La prophétie de Néferty », Supplément au Cahier Évangile, 89, p. 10-13.

Dieleman 2003 : J. Dieleman, «De profetie van Neferti », dans R.J. DemaréE, K.R. VEENHOF (éd.), Zij schreven geschiedenis: historische documente uit het Oude Nabije Oosten (2500-100 v. Chr.), Louvain, p. 73-79.

ENMARCH 2007 : R. ENMARCH, « What the Ancestors foretold: some References to Prediction in Middle Egyptian Texts », dans Th. SHNEIDER et K. SzPAKOwsKA (éd.), Egyptian Stories: a British Egyptological Tribute to Alan B. Lloyd on the Occasion of his Retirement, Münster, p. 75-86.

ENMARCH 2008 : R. ENMARCH, The Dialogue of Ipuwer and the Lord of All (Griffith Institute Publications), Oxford.

ERMAN 1911 : A. ERMAN, « Denksteine auf der thebanischen Gräberstadt », Sitzungsberichte der Königlich Preußischen Akademie der Wissenschaften, 49, p. 1086-1110.

ERMAN 1923 : A. ERMAn, Der Literatur der Aegypter, Leipzig.

FECHT 1972: G. FECHT, Der Vorwurf an Gott in den "Mahnworten des Ipu-wer" (Pap. Leiden I 344 recto, 11,11-13,8;15,13-17,3): zur geistigen Krise der ersten Zwischenzeit und ihrer Bewältigung, Heidelberg.

FELBER 2005 : H. FELBER, «"Ich werde dir zeigen, wie ein Sohn zum Gegner wird, ein Bruder zum Feind...": Konzepte von Gegnerschaft in literarischen Reden des Mittleren Reiches », dans H. FeLBER (éd.), Feinde und Aufrührer: Konzepte von Gegnerschaft in ägyptischen Texten besonders des Mittleren Reiches, Leipzig et Stuttgart, p. 62-73.

FOSTER 2001 : J.L. Foster, Ancient Egyptian Literature: An Anthology, Austin, p. 76-84. 
FÓTI 1976 : L. FÓTI, «The History in the Prophecies of Neferti: Relationship between the Egyptian Wisdom and Prophecy Literatures », dans L. KÁKOSY, E. GAÁL, Studia Aegyptiaca, 2, Budapest, p. 3-18.

FRANDSEN 2000 : P.J. FRANDSEN, « On the Origin of the Notion of Evil in Ancient Egypt », Göttinger Miszellen, 179, p. 9-34.

FRANDSEN 2003 : P.J. FRANDSEN, «Le fruit défendu dans 1'Égypte ancienne, Bulletin de la Société d'égyptologie de Genève, 25, 2002-2003, p. 57-74.

Franke 1994 : D. Franke, Das Heiligtum des Heqaib auf Elephantine: Geschichte eines Provinzheiligtums im Mittleren Reich (Studien zur Archäologie und Geschichte Altägyptens, 9), Heidelberg.

Gabolde 2015 : M. Gabolde, Toutankhamon (Les Grands Pharaons), Paris.

GARDINER 1905 : A.H. GARDINER, « Hymns to Amon from a Leiden papyrus », Zeitschrift für ägyptische Sprache und Altertumskunde, 42, p. 12-42.

GARDINER 1914 : A.H. GARDINER, «New Literary Works from Ancient Egypt: II. Pap. Petersburg 1116 B, recto », Journal of Egyptian Archaeology, 1, p. 100-106.

GARDINER 1953 : A.H. GARDINER, «The Coronation of King Haremhab», Journal of Egyptian Archaeology, 39, p. 13-31.

GieweKemeyer 2013 : A. GieweKeMEyer, «Perspektiven und Grenzen der Nutzung literarischer Texte als historische Quellen: zu Versuchen, "Geschichte" aus der Geschichte über die Vorhersagen des Neforti herauszulesen », dans G. MoERS, K. WIDMAIER, A. GIEWEKEMEYER (et al.) (éd.), Dating Egyptian Literary Texts, Hambourg, p. 285-365.

GNIRS 2006 : A.M. GNIRS, « Das Motiv des Bügerkriegs in Merikare und Neferti: zur Literatur der 18. Dynastie », dans G. Moers, H. Behlmer, K. Demuss et K. Widmaier (éd.), 'In.t dr.w: Festschrift für Friedrich Junge, vol. 1, Göttingen, p. 207-265.

Goedicke 1977 : H. Goedicke, The Protocol of Neferyt: the Prophecy of Neferti, Baltimore.

GOLÉNISCHEFF 1913 : W.S. GOLÉNISCHEFF, Les papyrus hiératiques $n^{o s} 1115,1116$ A et 1116 $B$ de l'Ermitage impérial à Saint-Pétersbourg, Saint-Pétersbourg.

Grandet 1994 : P. GRANDET, Le Papyrus Harris I (BM 9999), vol. 1 (Bibliothèque d'Étude de l'Institut Français d'Archéologie Orientale, 109.1), Le Caire.

GRAPOW 1931: H. GRAPOW, «Die Welt vor der Schöpfung (ein Beitrag zur Religionsgeschichte) », Zeitschrift für ägyptische Sprache und Altertumskunde, 67, p. 34-38.

GRIFFITH 1898 : F.L. GRIFFITH (éd.), The Petrie Papyri. Hieratic Papyri from Kahun and Gurob (principally of the Middle Kingdom), Londres.

GuILHOU 1986 : N. GuILHOU, « Réflexions sur la conception du mal à travers quelques grands mythes antiques », dans Hommages à François Daumas, vol. 2 (Orientalia Monspeliensia, 3), Montpellier, p. 361-371.

Guilhou 1989 : N. Guilhou, La vieillesse des dieux, Montpellier.

Guilhou 1998 : N. Guilhou, «Un nouveau fragment du Livre de la Vache Céleste », Bulletin de l'Institut français d'archéologie orientale, 98, p. 197-213.

Helck 1970: W. Helck, Die Prophezeiung des Nfrt.tj. Textzusammenstellung (Kleine ägyptische Texte, 2), Wiesbaden. 
HERRMANN 1957 : S. HERRMANN, Untersuchungen zur Überlieferungsgestalt mittelägyptischer Literaturwerke, Berlin.

Hornung 1979: E. Hornung, Das Totenbuch der Ägypter. Eingeleitet, übersetzt und erläutert (Bibliothek der alten Welt. Reihe der alte Orient), Zürich.

Hornung 1982 : E. Hornung, Der Ägyptische Mythos von der Himmelskuh: eine Ätiologie des Unvollkommenen, Fribourg.

KÁKOSY 1963 : L. KÁKOSY, « Schöpfung und Weltuntergang in der ägyptischen Religion », Acta antiqua, 11, p. 17-30.

KÁKosy 1964 : L. KÁKosy, «Ideas about the Fallen State of the World in the Egyptian Religion: Decline of the Golden Age », Acta orientalia, 17, p. 205-216.

KÁKosy 1992 : L. KÁKosy, « Hermes and Egypt», dans A.B. Lloyd (éd.), Studies in Pharaonic Religion and Society in Honour of J. Gwyn Griffiths (Egypt Exploration Society. Occasional Publications, 8), Londres, p. 258-261.

Kemboly 2010 : M. Kemboly, The Question of Evil in Ancient Egypt (Golden House Publications. Egyptology, 12), Londres.

KRI : K.A. KiTCHEN, Ramesside Inscriptions. Historical and Biographical, Oxford, 19821983.

Koenig 1994 : Y. Koenig, Magie et magiciens dans l'Égypte ancienne (Bibliothèque de l'Égypte ancienne), Paris.

LACAU P. 1909 : P. LACAU, Catalogue général des antiquités égyptiennes du Musée du Caire. $N^{o s}$ 34001-34064. Stèles du Nouvel Empire, Le Caire.

LACAU 1971 : P. LACAU, "Les verbes wbn, « poindre » et ps $\underline{d}$, « culminer»», Bulletin de l'Institut français d'archéologie orientale, 69, p. 1-9.

Lalouette 1984 : Cl. Lalouette, Textes sacrés et textes profanes de l'ancienne Égypte, vol. II. Mythes, contes et poésie (Connaissance de l'Orient, 63), Paris.

LANCZKOWSKi 1960 : G. LANCZKOWsKI, Altägyptischer Prophetismus (Ägyptologische Abhandlungen, 4), Wiesbaden.

LEFÈBVRE 1949 : G. LEFÈBVRE, Romans et contes égyptiens de l'époque pharaonique, Paris.

Lesko 2006 : L.H. LESKO, « The End is Near », dans K. SzPAKOWSKA (éd.), Through a Glass darkly: Magic, Dreams \& Prophecy in Ancient Egypt, Swansea, p. 63-70.

LEXA 1926 : F. LEXA, Papyrus Insinger : les enseignements moraux d'un scribe égyptien du premier siècle après J.-C., vol. 1. Texte, transcription, traduction et commentaire, Paris.

Lichtheim 1973 : M. Lichtheim, Ancient Egyptian Literature, vol. I. The Old and Middle Kingdoms, Berkeley et Londres.

Lichtheim 1980 : M. Lichtheim, Ancient Egyptian Literature, vol. III. The Late Period, Berkeley.

LOPRIENO 2003 : A. LOPRIENO, "Theodicy in Ancient Egyptian Texts », dans A. LAATO, J.C. DE MOOR (éd.), Theodicy in the World of the Bible, Leyde, p. 27-56.

LORTON 1993 : D. LORTON, "God's Beneficent Creation: Coffin Texts Spell 1130, the Instructions for Merikare, and the Great Hymn to the Aten », Studien zur altägyptischen Kultur, 20, p. 125-155. 
Mathieu 1993: B. MathieU, «Sur quelques ostraca hiératiques littéraires récemment publiés », Bulletin de l'Institut français d'archéologie orientale, 93, p. 335-347.

Meeks, Favard-Meeks 1995 : D. Meeks, Chr. Favard-Meeks, Les dieux égyptiens (La vie quotidienne), Paris.

MiCHEL 2014 : M. MiCHEL, Les mathématiques de l'Égypte ancienne. Numération, métrologie, arithmétique, géométrie et autres problèmes (Connaissance de l'Égypte ancienne, 12), Bruxelles.

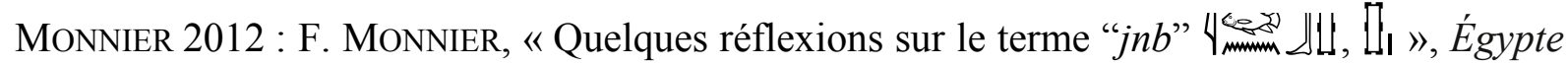
nilotique et méditerranéenne, 5, p. 257-283.

NaVILle 1886 : E. NAVILle, Das Ägyptische Totenbuch der XVIII. bis XX Dynastie, vol. 1. Text und Vignetten, Berlin.

Newberry 1893 : P.E. Newberry, Beni Hasan, vol. I (Archaelogical Survey of Egypt), Londres.

OBSOMER 1995 : Cl. OBSOMER, Sésostris I ${ }^{e r}$. Étude chronologique et historique du règne (Connaissance de l'Égypte ancienne, 5), Bruxelles.

OCKINGA 1988 : B. OcKIngA, « Totenbuch, Kapitel 175 », dans C. BuTTERwECK (éd.), Grab-, Sarg-, Votiv- und Bauinschriften, Texte aus der Umwelt des Alten Testaments, II/4, Gütersloh, p. 518-522.

Отто 1951 : Е. Отто, Der Vorwurf an Gott: Zur Entstehung der ägyptischen Auseinandersetzungsliteratur, Hildesheim.

Oтtо 1962 : E. Otто, «Zwei Paralleltexte zu TB $175 »$, Chronique d'Égypte, 37, p. 249-256.

PARKInSON 1991 : R.B. PARKInson, Voices from Ancient Egypt. An Anthology of Middle Kingdom Writings, Londres.

PARKINSON 1997a: R.B. PARKINSON, «The Text of Khakheperreseneb. New Readings of EA 5645, and an Unpublished Ostracon », Journal of Egyptian Archaeology, 83, p. 55-68.

PARKINSON 1997b: R.B. PARKInson, Tale of Sinuhe and Other Ancient Egyptian Poems: 1940-1640 BC, Oxford.

PARKINSON 1999 : R.B. PARKInSON, «Two New "Literary" Texts on a Second Intermediate Period Papyrus?», dans J. Assmann, E. Blumenthal (éd.), Literatur und Politik im pharaonischen und ptolemäischen Ägypten: Vorträge der Tagung zum Gedenken an Georges Posener, 5.-10. September 1996 in Leipzig, Le Caire, p. 177-196.

PARKInSON 2002 : R.B. PARKInSON, Poetry and Culture in Middle Kingdom Egypt. A Dark Side of Perfection, Londres et New York.

PARYs 2017 : L. PARYS, Le récit du Papyrus Westcar. Texte, traduction et interprétation (Textes égyptiens, 1), Bruxelles.

PÉREZ-ACCINO 2015 : J.R. PÉREZ-ACCINO, «Who is the Sage talking about? Neferty and the Egyptian Sense of History », dans P. Kousoulis, N. LAZARIDIS (éd.), Proceedings of the Tenth International Congress of Egyptologists: University of the Aegean, Rhodes. 22-29 May 2008 (Orientalia lovaniensia analecta, 241), vol. 2, Louvain, p. 1495-1502.

PoSENER 1956 : G. PoSENER, Littérature et politique dans l'Égypte de la XII e dynastie (Bibliothèque de l'École des Hautes Études. Sciences historiques et philologiques, 307), Paris. 
POSENER 1975 : G. POSENER, « La piété personnelle avant l'âge amarnien », Revue d'Égyptologie, 27, p. 195-210.

QUIRKE 2004 : S. QUIRKE, Egyptian Literature 1800 BC: Questions and Readings, Londres.

RoEDER 1927 : G. ROEDER, Altägyptische Erzählungen und Märchen, Iéna.

Rosmorduc 2009 : S. Rosmorduc, « De quelques passages de la Stèle d'Israël », Revue d'Égyptologie, 60, p. 139-146.

RYHOLt 1990 : K. RYHOLT, «A Reconsideration of some Royal Nomens of the Thirteenth Dynasty », Göttinger Miszellen, 119, p. 101-113.

Sandman 1938 : M. SAndman, Texts from the Time of Akhenaten (Bibliotheca Ægyptiaca, VIII), Bruxelles.

SAUNERON 1953: S. SAUNERON, «L'hymne au soleil levant des papyrus du Berlin 3050, 3056 et 3048 », Bulletin de l'Institut français d'archéologie orientale, 53, p. 65-90.

SAUNERON 1962: S. SAUNERON, Les fêtes religieuses d'Esna aux derniers siècles du paganisme (Publication de l'IFAO, Esna V), Le Caire.

SAuneron 1969 : S. SAuneron, Le temple d'Esna, vol. IV. Nos 399-472 (Publication de l'IFAO, Esna IV), Le Caire.

SCHENKEL 1984 : W. SCHENKEL, « Sonst-Jetzt: Variationen eines Literarischen Formelements », Die Welt des Orients, 15, p. 51-61.

SEIBERT 1967 : P. SEIBERT, Die Charakteristik: Untersuchungen zu einer altägyptischen Sprechsitte und ihren Ausprägungen in Folklore und Literatur, 1. Philologische Bearbeitung der Bezeugungen (Ägyptologische Abhandlungen, 17), Wiesbaden.

SHUPAK 2006 : N. SHUPAK, "The Egyptian "Prophecy": a Reconsideration », dans K. ZIBELIUS-CHEN, H.-W. FISCHER-ELFERT (éd.), "Von reichlich ägyptischem Verstande": Festschrift für Waltraud Guglielmi zum 65. Geburtstag, Wiesbaden, p. 133-144.

SIMPSON 1973 : W.K. SIMPSON (éd.), The Literature of Ancient Egypt: an Anthology of Stories, Instructions, and Poetry, New Haven.

SitZler 1995: D. SitZleR, Vorwurf gegen Gott. Ein religiöses Motiv im Alten Orient (Ägypten und Mesopotamien) (Studies in Oriental Religions, 32), Wiesbaden.

Sмith 1994 : H. SMIth, « Ma'et and Isfet», The Bulletin of the Australian Centre for Egyptology 5, p. 67-88.

Smith 2000 : H. SMith, «P. Carlsberg 462. A Fragmentary Account of a Rebellion against the Sun God», dans P.J. Frandsen, K. Ryholt (éd.), The Carlsberg Papyri 3. A Miscellany of Demotic Texts and Studies, Copenhague, p. 95-112.

Stadler 2009 : M.A. Stadler, Weiser und Wesir. Studien zu Vorkommen, Rolle und Wesen des Gottes Thot im ägyptischen Totenbuch, vol. 1 (Orientalische Religionen in der Antike, 1), Tübingen.

StAUder 2013 : A. STAUDER, Linguistic Dating of Middle Egyptian Literary Texts (Lingua aegyptia. Studia Monographica, 12), Hambourg.

STRUVE 1925 : V.V. STRUVE, « Le papyrus 1116r et la littérature prophétique de l'ancienne Égypte », Zopiski Kollegii Vostokovedov, 1, p. 209-227. 
TARASENKO 2016a: M. TARASENKO, «The "Children of Nūt" and their Rebellion in Chapter $175^{\mathrm{A}}$ of the Book of the Dead», dans N. Guilhou (éd.), Liber amicorum - speculum siderum : Nüt Astrophoros, Papers presented to Alicia Maravelia, Oxford, p. 35-48.

TARASENKo 2016b: M. TARASENKO, Studies on the Vignettes from Chapter 17 of the Book of the Dead, 1. The Image of mś.w Bdšt in Ancient Egyptian Mythology (Archaeopress Egyptology, 16), Oxford.

TARASEnKo 2017 : M. TARASENKO, " "Children of Weakness" in the Book of Gates », dans M. TOMORAD, J. Popielska-GrZyBowska (éd.), Egypt 2015: Perspectives of Research. Proceedigs of the Seventh European Conference of Egyptologists, $2^{\text {nd }}-7^{\text {th }}$ June 2015, Zagreb, Oxford, p. 31-43.

Urk. IV : K. SeTHE, Urkunden der 18. Dynastie, vol. 1 (Urkunden des ägyptischen Altertums, IV), Leipzig, 1906.

Urk. VII : K. SETHE, Historisch-biographische Urkunden des Mittleren Reiches (Urkunden des ägyptischen Altertums, VII), Leipzig, 1935.

VANDIER 1936 : J. VANDIER, La famine dans l'Égypte ancienne, Le Caire.

VARILLE 1941 : A. VARILLE, «L'hymne au soleil des architectes d'Aménophis III Souti et Hor », Bulletin de l'Institut français d'archéologie orientale, 41, p. 25-30.

VERHOEVEN 2013 : U. VERHOEVEN, «Literatur im Grab - der Sonderfall Assiut», dans G. Moers, K. Widmaier, A. GieweKemeyer (et al.) (éd.), Dating Egyptian Literary Texts, Hambourg, p. 139-158.

VERNUS 1995 : P. VERNUS, Essai sur la conscience de l'histoire dans l'Égypte pharaonique (Bibliothèque de l'École des hautes études, sciences historiques et philologiques, 332), Paris.

VON BECKERATH 1984: J. VON BECKERATH, Handbuch der Ägyptischen Königsnamen (Münchner Ägyptologische Studien, 49), Munich.

WESTENDORF 1986a: W. WESTENDORF, «Einst-Jetzt-Einst oder: die Rückkehr zum Ursprung », Die Welt des Orients, 17, p. 5-8.

WeStendORF 1986b: W. WestendORF, «Theodizee », dans Lexikon der Ägyptologie, VI, col. 473.

Yoyotte 1962 : J. YoyotTe, «Processions géographiques mentionnant le Fayoum et ses localités », Bulletin de l'Institut français d'archéologie orientale, 61, p. 79-138. 